\title{
Corela
}

Cognition, représentation, langage

17-1 | 2019

Vol. $17, \mathrm{n}^{\circ} 1$

\section{UP à contre-sens}

\section{Catherine Douay et Daniel Roulland}

\section{OpenEdition}

\section{Journals}

Édition électronique

URL : http://journals.openedition.org/corela/8037

DOI : $10.4000 /$ corela.8037

ISSN : 1638-573X

\section{Éditeur}

Cercle linguistique du Centre et de l'Ouest - CerLICO

\section{Référence électronique}

Catherine Douay et Daniel Roulland, «UP à contre-sens », Corela [En ligne], 17-1 | 2019, mis en ligne le 17 juin 2019, consulté le 01 juillet 2019. URL : http://journals.openedition.org/corela/8037 ; DOI : $10.4000 /$ corela.8037

Ce document a été généré automatiquement le 1 juillet 2019.

\section{(c) (i) (2)(2)}

Corela - cognition, représentation, langage est mis à disposition selon les termes de la licence Creative Commons Attribution - Pas d'Utilisation Commerciale - Partage dans les Mêmes Conditions 4.0 International. 


\title{
UP à contre-sens
}

\author{
Catherine Douay et Daniel Roulland
}

'C'est parce que le sens se constitue et émerge en tant que tel dans un acte de reconnaissance commun - ou dans un acte de communion - qu'il peut avoir une existence objectivement connaissable à deux êtres humains' (Larsson 1997 :

80). On sort par là-même de l'impasse à laquelle semble nous condamner la conception classique $\mathrm{du}$ sens : le sens n'est pas objectif au sens où il renvoie

à des entités existant objectivement indépendamment du langage et des êtres humains, mais il n'est pas subjectif pour autant non plus. Le fait d'être un phénomène émergeant intersubjectivement lui assure un statut qui évite les difficultés souvent débattues en philosophie qui découlent des positions opposées de l'objectivisme

et du subjectivisme.

(G. Kleiber 2001 : 22, citant Larsson 1997)

The ultimate basis of speech is the fact that individual thoughts and feelings are, as such, entirely inalienable. One man cannot think the thoughts of another, or behold an object with another's mental vision. Nor can anyone take his enjoyment of a sunset and transfer it directly to a companion's mind. It is the penalty of individuality that the inner life is solitary, that perceptions and feelings cannot actually be shared. (A.H. Gardiner 1932 : §23, 67) 


\section{Introduction $^{1}$ : dans quel sens prendre UP ?}

1 Dans l'ensemble des particules ${ }^{2}$ en anglais, UP présente des caractéristiques remarquables, dont en particulier une fréquence et une variété d'usage ${ }^{3}$ considérables, ce qui explique que les regroupements de ses emplois sous dénominateur commun sont amenés à annexer diverses valeurs inclassables, souvent en proportion importante. Malgré tout, dans le but d'en faciliter la description ou l'apprentissage, les manuels proposent régulièrement une définition générale unique, de type spatial, impliquant un « mouvement vertical vers le haut ». C'est ce type de définition qui est indiqué en premier dans les dictionnaires. C'est aussi cette définition qui est couramment donnée comme la plus «évidente »: l'association de UP avec un mouvement vertical vers le haut parait "tomber sous le sens ». Or, la "sémantique de l'évidence», pour reprendre l'heureuse formule de P. Jalenques (2002 : 74-90), n'a jamais été un gage de fiabilité. En réalité, faire appel à l'évidence permet opportunément de ne pas interroger les rapports complexes entre le langage et l'espace physique. Si c'était une relation spatiale qui était déterminante dans la valeur de UP, l'évidence reviendrait à accorder la priorité à une référence en termes de "pointage des objets du monde ", ce que dénonce par exemple à juste titre P. Laurendeau (1998), en rappelant la position très nette d'A. Culioli à ce propos:

Pour le linguiste, c'est une catastrophe. Le problème de la référence au monde ne le concerne pas. C'est la conséquence du fait que nous ne travaillons pas de façon strictement extensionnelle. Nous [linguistes - P.L.] travaillons sur des propriétés, sur des objets que nous [nous énonciateurs, cette fois-ci !- P.L.] construisons. De ce point de vue, le problème de la référence doit être écarté. (Culioli 1985: 82, cité par Laurendeau 1998 : \$19)

2 Ceci dit, l'interprétation en termes spatiaux peut être étayée par de très nombreux exemples (get up « se lever », go up « monter », etc). L'association de UP avec le « haut », au contraire de DOWN qui est associé au «bas", n'est pas discutable. Si donc nous refusions la détermination première de l'espace, en raison du référentialisme qu'elle implique ("pointage des objets du monde»), nous devrions en contrepartie expliquer comment un système linguistique peut structurer l'espace en une relation de type UP / DOWN, et sur quelle base qui serait linguistique et qui ne serait pas référentiellement spatiale au départ. Nous pensons qu'approcher la question en rendant le spatial dépendant du linguistique, et non l'inverse, permet d'expliquer l'ensemble des cas d'emploi de UP. Autrement dit, la structuration de l'espace (en haut, en bas etc) est pour nous un des divers résultats d'une activité systémique d'un niveau beaucoup plus profond. Quand un énonciateur (que nous appelons pour notre part locuteur) structure l'espace autour de lui, c'est grâce à l'activité du système du langage dont il n'est que très partiellement conscient et sans laquelle il serait incapable d'opérer les distinctions et comparaisons nécessaires.

3 La thèse de la détermination spatiale laisse de très nombreuses valeurs de côté et promeut indûment les cas spatiaux qui, si on s'en réfère aux constructions, sont très fréquents dans les corpus mais structurellement minoritaires. Par exemple, l'association de UP à la notion d'un mouvement vers le haut devrait en rendre l'emploi incompatible avec des verbes impliquant un mouvement de descente. Or on a volontiers swallow up ou gulp up au sens d'«avaler» ou «engloutir». C'est donc que quelque autre composant 
sémantique ou pragmatique intervient, mais on voit qu'on n'est plus du tout dans le domaine spatial.

4 On a aussi recours, pour expliquer les emplois « déviants ", à une forme ou une autre de métaphorisation: par exemple, on associera UP à une notion d'augmentation par opposition à une diminution qui serait exprimée par DOWN (Prices are up vs down «Les prix montent » vs « baissent »). Mais c'est une sorte de fuite en avant qui s'annonce, car cette solution pose un nouveau problème : celui de la métaphorisation elle-même, qu'on ne peut pas se contenter de tenir pour évidente. D'autre part, la langue semble se jouer du chercheur et toute solution trop rapide se voit immédiatement contredite. Par exemple, slow down $n^{5}$ est donné par les dictionnaires comme signifiant un ralentissement, ce qui est évidemment compatible avec le sens réputé canonique de diminution et d'abaissement de DOWN, mais aussitôt, au détour d'un texte ou d'une conversation, apparaît slow up avec le même sens. Que faut-il en conclure ? Sans doute que le véritable sens de UP ou de DOWN est beaucoup moins immédiat que ne le sont les relations spatiales ou quantitatives de type « abaissement » et « diminution ». C'est la même chose pour let up qui peut signifier une diminution au sens propre (bulletin météo: The snow shows no sign of letting up " Aucun signe de ralentissement / d'accalmie côté neige »), comme au sens figuré (There should be no let up in prevention "Il ne devrait y avoir aucun relâchement en matière de prévention »).

5 Sans trop s'avancer dans la caractérisation, on voit que ces valeurs, qui dévient du modèle spatialiste, font prévaloir autre chose qu'une structuration spatiale basique. La question devient dès lors de savoir ce qu'est cette " autre chose ». C'est cela qu'il faut découvrir en linguistique. Si, comme on l'observe, cette « autre chose » est constamment observable, il n'y a aucune raison de la tenir pour une déviance ou une perturbation. Elle peut être au contraire posée d'emblée comme une forme vectrice et structurante. Cela évitera de devoir postuler que l'espace est une sorte d'absolu fondateur. A tout le moins, les valeurs spatiales et les valeurs non spatiales, qui manifestent cette "autre chose » qui est en cause ici (la dimension proprement linguistique), coexistent en permanence. Elles sont constamment mêlées et ont toujours coexisté6.

6 Si le spatial n'est pas déterminant, alors il faut chercher dans l'ordre linguistique luimême les motifs réels de sa structuration. La critique de la primarité du spatial a bien entendu déjà été faite. Parmi ces critiques, nous retenons l'analyse de P. Cadiot (2002) qui critique la notion de préposition spatiale selon les quatre aspects ou «dimensions » de la question: les dimensions psychologique (l'espace tenu pour l'ancrage de base pour la parole), génétique (de quel espace est-il question? autocentré ? géométrique ? l'espace concret des matérialités ? les rapports différentiels aux objets ?), grammaticale (de quel sens est-il question? le sens en contexte et hors contexte, le sens " anthropique ", le sens lexical ou phrastique, la prototypie de binarismes antonymiques ?), et étymologique (sens non spatiaux et spatiaux comme contemporains) :

La leçon générale, évidente, de tout cela, c'est que ce qu'il y a de commun aux exemples spatiaux est aussi - exactement - ce qu'ils partagent avec les sens non spatiaux (2002:127).

7 P. Cadiot explique qu'on retrouve les mêmes composants relationnels dans les emplois spatiaux et non spatiaux, ce qui fait pencher la balance vers les interprétations relationnelles de niveau plus opératoire. Il estime qu'on ne peut même pas légitimement isoler des emplois spatiaux convaincants, y compris en inventant des continuums qui moduleraient les valeurs d'un « plus spatial » vers un « moins $»^{7}(2002: 119)$. 
Une optique plus cognitive amène à privilégier la métaphorisation, comme nous l'avons esquissé plus haut. Les constructions linguistiques refléteraient un processus de métaphorisation qui tiendrait à l'action de l'organisme. C'est ainsi que Vandeloise (1987) entend corriger le modèle spatial simple en posant un principe d'anticipation fondé par une « recherche » du locuteur $(1987: 110)$ :

Selon ce principe, les prépositions qui introduisent une relation entre un site et une cible statique introduisent la même relation entre un site et le terme anticipé du déplacement d'une cible mobile (c'est nous qui soulignons).

Dans le modèle de Lakoff \& Johnson (1985), Lakoff (1987), la métaphore s'observe dans la pensée et dans l'action, avant de se manifester dans le langage, ce qui revient à autonomiser un système conceptuel, indépendamment de la langue. C'est ce qu'observent justement H. Constantin de Chanay \& S. Rémi-Giraud (2002) :

Rhétoriquement parlant, cela revient à donner aux transferts métaphoriques des assises métonymiques ou synecdochiques qui en redoublent la motivation; et cognitivement parlant, à fonder les structures sémantiques sur celles du "réel », non certes celui de la physique, mais tout de même celui du "vécu». Les métaphores spatiales sont au cœur du débat dans la mesure où elles manifestent que nous appréhendons des abstractions à travers des représentations concrètes liées à la vue, à la localisation et aux déplacements, et elles sont à ce titre le fer de lance de ces conceptions «expérientialistes » qui considèrent tout concept comme « embodied» (2002: 78).

Cette conception « expérientialiste » implique plusieurs propositions problématiques. La première est que l'espace en jeu dans la métaphore ne peut pas être limité à celui de l'expérience concrète et donc doit être « représenté », pour prêter sa « matérialité » à des « découpages signifiants, c'est-à-dire un réservoir de signifiants disponibles, condition $a$ priori de tout phénomène d'intellection" (Constantin de Chanay \& Rémi-Giraud, 2002 : 79). Or « intellection » et « représentation » ne sont pas définis, ce qui fait encore une fois reculer le problème sans le résoudre. La seconde difficulté est que le langage perd toute capacité structurante et les auteurs le définissent comme un "medium de répercussion parmi d'autres possibles». Nous retrouvons alors, en décalage mais avec une acuité renouvelée une problématique référentialiste: la métaphore suppose un système conceptuel indépendant du langage, donc traitant plus directement que lui de la référence. La métaphorisation ne fournit pas malheureusement l'assise ou l'explication de ce traitement et s'avère contrainte d'en rendre le périmètre singulièrement flou.

11 Troisème point : l'une et l'autre version du référentialisme (celui du « réel physique » et celui du "réel vécu») ne rendent pas compte de la variabilité des métaphores selon les diverses langues du monde, mais également pour une langue donnée. Prenons l'exemple de l'expression anglaise cheer somebody up « remonter le moral à quelqu'un». Le sens réputé premier de «montée » y serait métaphoriquement développé dans le sens d'une élévation figurée du moral. La "sémantique de l'évidence » a beau jeu d'expliquer qu'en français aussi on "remonte le moral» comme on " (re)monte 8 la pente ». Mais dans un exemple comme Somebody has messed everything up again!, on pourrait avoir au choix "Quelqu'un a encore tout fichu en l'air », aussi bien que " tout fichu par terre ", avec un mouvement orientable dans les deux sens. De même pour give up! qu'on peut traduire par «baisser les bras » ou « laisser tomber». Il y a aussi des cas où le français «(re)monter » se traduirait en anglais par un verbe ou un nom composé avec DOWN, diamétralement opposé à UP : par exemple «Le patron lui a remonté les bretelles » peut se traduire par The boss gave him a good dressing-down. 
12 La traduction est ici révélatrice : en réalité, UP se rencontre aussi bien dans des cas de mouvement vers le haut que vers le bas (au sens propre ou figuré), vers l'arrière comme dans back up "faire marche arrière " ${ }^{9}$ ou vers l'avant comme dans get up front "aller à l'avant (d'un véhicule) » ou move up « avancer ». UP peut aussi et surtout n'évoquer aucun mouvement dans une direction quelconque.

13 Dans de nombreux cas, les linguistes sont contraints d'avouer que UP n'a plus aucun lien avec ce qui leur avait semblé évident au départ et il les reléguent dans la catégorie « autres cas » ou « sens non prévisibles ». H. Adamczewski (1992), par exemple, distingue les situations où UP «apporte une précision facilement déchiffrable » (sens de mouvement vers le haut) de celles où « la particule modifie le sens du verbe initial de façon non prévisible». Il écrit: "Dans Put up your hands (Levez la main!) up est transparent : ce n'est hélas (sic) plus le cas dans: We can put you up when you come to Paris ${ }^{10}$ (On peut vous loger). De même look up (lever les yeux) est clair mais look up a word (chercher un mot dans le dictionnaire) l'est beaucoup moins » (1992 : 158-159).

14 Une alternative à la conception spatiale, qui a l'avantage de se présenter comme une relation proprement linguistique est la définition de UP en termes d'aspect. Il s'agirait d'un marqueur d'aspect perfectivant au sens où la particule signifierait la complétude de l'événement dénoté par le verbe lexical, sous la forme d'un résultat, atteint ou non, mais en tout cas visé ${ }^{11}$. C'est ce sens de complétude, souvent associé à celui de télicité ${ }^{12}$, qui pour D. Denison (1985) constitue le sens fondamental de UP, dont dérivent toutes les interprétations possibles :

One of the most general definitions [of up in the OED] is 'to or towards a state of completion or finality. (Frequently serving merely to emphasize the import of the verb)' (...), and many of the other senses can be seen as variants of this completive or intensive sense. Accordingly, I shall use the term 'completive up' as a shorthand label for a whole nexus of Aktionsart values of up, possibly incorporating other idiomatic nuances of meaning but not including a component of meaning which is spatial or transparently derived from a spatial sense (Denison $1985: 2$ ).

[L'une des définitions les plus générales [de up dans l'OED] est « jusqu'à, ou vers, un état de complétude ou de finalité. (Souvent utilisé simplement pour renforcer l'apport du verbe)", et beaucoup des autres sens peuvent être vus comme des variations de ce sens de complétude ou d'intensité. Par conséquent, j'emploierai l'expression « up de complétude » comme raccourci pour désigner tout un ensemble de valeurs aspectuelles de up, en incorporant potentiellement d'autres nuances de sens idiomatiques, mais qui n'incluent pas une composante de sens spatial ou dérivé de façon transparente d'un sens spatial.]

15 Pour illustrer cette valeur de «completive up», Denison propose Eat up! «Mange (+UP)!», qui est l'exemple le plus fréquemment repris par les dictionnaires et grammaires. Il précise : «Up imparts perfectivity or completion or exhaustiveness » [UP confère un sens de perfectivité ou d'achèvement ou de complétude]. La traduction française, qui est invariablement du type «Finis ton assiette ! " semble, comme c'était le cas pour «monter» ou «(se) lever » dans l'interprétation spatiale, corroborer le bien fondé de cette thèse aspectuelle. Cependant, Denison lui-même reconnaît qu'il est très difficile de rattacher tous les emplois figurés à l'aspectualité, tant leur variété est grande. UP en vient à exprimer, dit-il, des sens hautement divergents, voire contradictoires :

It is very difficult to classify all of its figurative uses, 'the variety of which is so great that the adverb comes to present a number of highly divergent and even directly opposite senses, e.g. to bind up... in contrast with to break up' (OED s.v. up adv. ${ }^{1}$ II, [p.38] note). In the case of bind up and break up I would suggest that the 'directly opposite senses' have more to do with bind and break than with up, and in 
fact the real basis of much of OED's classification is (very properly) the different groups of verbs with which up collocates rather than the meaning of up itself. (1985:2)

[Il est très difficile de classer tous les emplois figurés de up dont la variété est si grande que l'adverbe en vient à présenter un grand nombre de sens très divergents, voire directement opposés, comme dans, par exemple, to bind up (relier) par contraste avec to break up (rompre) (OED s.v. up adv. ${ }^{1}$ II, [p.38] note). Dans le cas de bind up et break up, je pense que les "sens directement opposés " concernent davantage bind et break que up et qu'en fait une grande partie de la classification de l'OED se base (à juste titre) sur les différents groupes de verbes avec lesquels up entre en collocation plutôt que sur le sens de up lui-même.]

On ne saurait dire plus clairement que la particule n'a finalement pas de sens précis, l'essentiel étant exprimé par le verbe lexical auquel elle est associée. Autrement dit, dans certains cas, la particule donnerait une indication aspectuelle déterminante, mais dans d'autres, elle viendrait simplement « compléter » le verbe, sans qu'on sache exactement à quel niveau (syntaxique ou sémantique), ni pourquoi le verbe anglais aurait besoin dans certains cas d'être « complété ». La classification de référence de l'OED est exclusivement basée sur le verbe, et Denison la juge simplement « tout-à-fait appropriée ${ }^{13}$ ».

Cette thèse de la particule qui viendrait simplement compléter ou renforcer le sens du verbe (voire serait redondante par rapport à lui) n'est pas de nature à clarifier la question. Dans une collocation telle que slow up (ralentir), on ne peut pas dire que UP ne fait que renforcer le sens du verbe. Quelle serait alors la différence entre slow up et slow down? Et pourquoi avons-nous UP, et non pas IN ou OFF ? Par ailleurs, comment pourraiton expliquer le fait très remarquable en anglais que le verbe lexical est volontiers peu explicite, et que c'est la particule qui donne le sens final de l'expression, comme c'est le cas pour get avec up, down, in, out, on, off, etc ? C'est davantage get qui est la variable, ainsi qu'on peut le constater en comparant get up « monter » avec des équivalents comme walk up, run up, rush up, rise up ou climb up, etc, où c'est UP qui est le véritable pivot de la construction: le verbe lexical qualifie une modalité d'action, une manière pour le sujet d'être sujet de l'action et non l'action elle-même dans sa portée effective. Ces collocations montrent que la particule a son plan propre de fonctionnement, distinct de celui du verbe, qu'elle ne se contente pas de compléter. La thèse de Denison suppose une continuité lexicale avec le verbe: or, si cette continuité était la clé de ces constructions, en toute logique c'est seulement DOWN qui devrait être associée à slow, et il existe de multiples contre-exemples similaires.

18 Nous entendons donc montrer que les particules ont leur systématique propre. On peut même considérer que c'est UP qui est le pivot de la collocation verbe + particule, et de son interprétation. C'est pour nous la particule qu'il convient de prendre prioritairement en considération. Comme nous le verrons, dans put up ou set up, par exemple, c'est UP qui est le vecteur principal de la signification et non le verbe lexical put ou set. Cela ne signifie pas, bien entendu, que l'apport du verbe lexical soit mineur. Le verbe porte les marques formelles de la prédication et énonce pour le sujet une manière d'être et d'agir sans laquelle le procès ne pourrait pas être construit. Mais si nous comparons par exemple abandon et give up, de même sens ("abandonner »), nous constatons que le phrasal verb distingue dans le verbe deux éléments prédicatifs, un élément verbal de type notionnel (le verbe lexical) et un second élément dont la nature et la fonction restent à élucider, alors que le verbe abandon, emprunté au roman, ne montre pas cette distinction et fusionne formellement ces deux éléments. C'est dans cette différence très importante de construction qu'il faut chercher la raison profonde du maintien de très nombreux 
doublons, généralement romans, face aux phrasal verbs (comparer abandon, quit / give up; commence, begin, start / take up; arrange / set up; raise / bring up; tolerate / put up (with); arrive/ turn up; etc). Ces doublons sont traditionnellement considérés comme des variantes de registre historiquement motivées ${ }^{14}$ alors qu'il s'agit en réalité d'un fait de structure de premier ordre qui n'a à ce jour pas reçu d'explication.

La thèse aspectuelle a été appliquée à toutes les particules, et excessivement étendue. A propos des langues germaniques, A. McIntyre (2003) $)^{15} \mathrm{a}$ pu parler de la télicité au sens où un résultat serait atteint comme d'un «mythe ». Ajutons que, si la dimension aspectuelle était primordiale, nous n'aurions plus de critères pour distinguer les particules entre elles, comme, par exemple, dans close up et close down (fermer), ou slow up et slow down (ralentir). On ne voit pas non plus ce qui différencierait use up (utiliser) ou end up (terminer) de use et end sans la particule. Ceci dit, c'est un fait que la particule en général apporte une dimension en quelque sorte «applicative » qui «finit» l'expression: si on compare walk avec walk in, walk off ou walk up ou down, on voit nettement que la particule précise une circonstance d'application du procès qui s'apparente à de l'aspectualité. Mais dans ce cas, nous pouvons affirmer que cette aspectualité est valable pour toutes les particules, et non pas seulement pour UP. On la retrouvera donc toujours dans toutes les constructions verbales à particule associée. C'est ce que confirme McIntyre (2003).

Restent enfin et surtout les cas d'emplois de UP - les plus nombreux - impossibles à interpréter ni dans un sens spatial ni dans un sens aspectuel. Ajoutons à ceux que nous avons déjà mentionnés des emplois courants comme :

1. What's up ? «Que se passe-t-il ?» / «Qu'est-ce qui ne va pas?»

2. The dishwasher is playing up again! « Le lave-vaisselle fait encore des siennes »

3. We have a good relationship but sometimes I feel like he babies me up too much «On

s'entend bien mais j'ai parfois le sentiment qu'il m'infantilise trop ${ }^{16} /$ me traite trop comme un bébé"

21 Selon la thèse que nous défendrons ici, la difficulté qu'on a à fournir une explication unitaire des effets de sens de UP vient du préjugé selon lequel le langage devrait au moins une partie de ses structures ou de leur valeur, sinon la totalité, au monde extérieur, qu'il soit reflété, copié, représenté ou référencié, selon le degré d'investissement qu'on accordera au langage dans l'opération ${ }^{17}$. Au minimum, nous pouvons considérer que le langage et le monde se présentent toujours mêlés pour le linguiste, mais si c'est le cas, rien ne permet d'affirmer que des représentations fonctionnent indépendamment du langage, avant ou sans lui. Et, par «langage » nous entendons la langue, le système du langage et non pas l'énonciateur qui l'utilise, et qui, lui, est précisément le creuset où les deux plans se mêlent. En tout état de cause, ce mélange intime et universel impose à l'analyste de refuser a priori toute forme de référentialisme externe et de tenir prudemment le système du langage - comme la théorie générale des systèmes l'exige d'ailleurs - pour prioritairement auto- ou sui-référentiel. Il ne doit sa structure qu'à sa nature propre de système. Par conséquent, UP est d'abord un élément linguistique et c'est d'abord la langue qui en détermine l'importance et la valeur.

L'étude qui suit traite seulement du sémantisme de UP et de sa place dans une systémique interlocutive. Elle n'aborde pas la question du statut catégoriel et syntaxique de la particule qui soulève de multiples problèmes à ce jour sans solution. Pour notre part, nous estimons que comprendre le sémantisme exact de la particule est la condition nécessaire, indispensable, à l'investigation syntaxique. 
ne première partie, nous montrerons le besoin d'une logique communicationnelle capable d'intégrer et de dépasser le modèle spatial dans un fonctionnement systémique (partie 1). Nous montrerons comment ce fonctionnement auto-référentiel complexe repose sur une triangulation interlocutive déterminant 3 «configurations» (partie 2). Nous expliquerons ainsi pourquoi la particule UP, dans son rapport oppositionnel à DOWN, doit être définie en configuration 1 (C1), différentielle, fortement sélective et distinctive, déterminant des valeurs "à contre-courant» par rapport à ce qui vaut $a$ contrario comme «norme » linguistique. Ceci posé, nous explorerons les emplois discursifs de UP (partie 3).

\section{Logique spatiale vs logique communicationnelle}

La logique spatiale a la faveur de nombreux linguistes, chez les diachroniciens (voir note 5) mais aussi chez les cognitivistes qui ont le souci de "naturaliser» les structures linguistiques autant que possible. Selon leur point de vue, l'espace n'est pas à prendre tel quel, de manière qu'on peut appeler "objective", mais il serait "subjectivisé » ou « anthropologisé » dans et par la relation de l'organisme à l'environnement. Pour autant, ce type d'approche qui récuse explicitement la primarité / primauté de l'espace objectif au profit d'un espace subjectivisé, ne parvient pas à s'affranchir totalement de la logique spatiale élémentaire. J-R. Lapaire \& W. Rotgé (1993) résument parfaitement la situation (Essai 5, Le vide et le plein dans l'étude du langage, 92-93):

En parlant du langage avec des termes spatiaux, nous lui imposons une logique spatiale, souvent à notre insu [...] Le fait le plus troublant et peut-être le plus mortifiant pour nous linguistes est que nous nous vautrons beaucoup plus dans la concrétude que nous ne le pensons. Alors que nous croyons nous élever vers les sphères d'une intellectualisation salutaire, nos pieds restent accrochés à la terre. En grattant la surface de nos concepts linguistiques les plus abstraits, nous retrouvons la force de la gravité, le monde de la matière, pour ne pas dire les jeux de cubes, de cailloux, de sable, du territoire de l'enfance.

La place de l'espace dans la langue, soulignent de leur côté Fagard \& Stocik (2012 : \$1), est régulièrement mise en avant par différentes théories « localistes ». Il est « souvent, sinon toujours, possible d'utiliser un terme spatial avec un sens métaphorique » (2012: \$10), ce qui fait qu'il est difficile de trouver des marques linguistiques "purement spatiales", mais nous pouvons ajouter: ce qui fait que l'espace est très aisément convocable pour illustrer des relations de manière simple. On en arrive à tenir ce qui est simple pour ce qui est premier et Lyons $(1980: 338)^{18}$ résume la thèse générale localiste ainsi :

Les expressions spatiales sont plus fondamentales, grammaticalement et lexicalement, que diverses espèces d'expressions non spatiales.

La primauté du spatial est particulièrement mise en avant dans les études sur l'apprentissage et l'acquisition de la langue maternelle: les premières constructions linguistiques de l'enfant concernent le mouvement et l'espace. Dans son étude attentive, E. Riguel (2015) montre ainsi parfaitement comment les phrasal verbs s'acquièrent chez les enfants anglophones par des usages holophrastiques des particules avec des valeurs spatiales (Up daddy! dit un enfant pour demander à son père de le prendre dans ses bras, et Train down pour le train-jouet qui est tombé au sol). Cela, pour l'auteure, confirme que le repérage spatial est « cognitivement plus simple » $(2015$ : note 10) et donne une assiette empirique à la triade énonciativiste du ego, hic et nunc, source des repérages spatiotemporels (2015: note 9). Selon elle, le monde spatial a donc un «statut privilégié et 
crucial pour la cognition, ainsi que pour la construction du langage en acquisition du langage» (2015: \$5.2). Que le mouvement soit crucial dans la mise en place de la cognition, c'est une chose, mais il convient de se méfier d'une confusion avec le « monde spatial». Au contraire, le mouvement est justement ce qui se distingue dans l'environnement spatial et permet de le structurer. Le fait que les particules soient utilisées comme des holophrases, puis comme des pivots, paraît au contraire selon nous en démontrer la saillance linguistique et dialogique pour l'enfant.

Dans l'hypothèse localiste que Fagard \& Stocik (2012) qualifient de «modérée ", il s'agit d'avoir recours à l'espace pour régler certaines conceptualisations délicates. On peut penser au temps, par exemple, qui ne peut pas être conceptualisé sans passer par des métaphores spatiales ${ }^{19}$. Mais l'hypothèse "forte " va jusqu'à défendre l'idée que les fondements de toutes les catégories grammaticales sont spatiaux. Par exemple, pour l'anglais, la périphrase be going to $V$ sera couramment analysée comme le produit d'une "grammaticalisation" de relations spatiales, qui affecterait de façon très générale les verbes dits "de déplacement». On passerait d'un mouvement physique concret à un mouvement abstrait vers le futur, en mélangeant au passage des éléments spatiaux et des éléments temporels dans une notion assez floue de «mouvement». Marquant initialement un déplacement dans l'espace (à partir d'un point ou vers un point, ou d'un point à un autre), ces verbes en seraient venus, diachroniquement, à marquer un renvoi à l'avenir. A propos de be going to, J-R. Lapaire et W. Rotgé (2004 : 225) expliquent ainsi que « le sujet cognitif voyage mentalement vers l'événement qu'il voit droit là-bas au bout du chemin ». Pour les théories de la grammaticalisation, c'est l'espace et l'expression des relations spatiales qui est historiquement et universellement au fondement de l'évolution sémantique des formes linguistiques. On voit que "grammaticalisation » signifie en fait " métaphorisation ${ }^{20}$ ", ce qui revient finalement à dire que tous les usages grammaticaux remontent à la seule et unique racine du déplacement spatial.

Les sémantiques cognitives ont par conséquent tendance à faire de la cognition spatiale (le traitement cognitif de l'espace) le pivot de la conceptualisation et donc, pour elles, de la structuration du signifié linguistique. Space grammar ("grammaire spatiale », voir Fortis 2010, Lapaire 2017) est d'ailleurs le premier nom que R. Langacker a donné à son modèle cognitif $(1982,2008)$. Certes, comme le précisent Fagard et Stocik (2012), les théories localistes ne se distinguent pas seulement par le degré d'importance qu'elles accordent à la place de l'espace en grammaire mais aussi et surtout par leur conception de l'espace lui-même. Entendue d'abord au sens étroit d'« espace physique », la notion a ensuite été élargie de plusieurs façons. Pour Langacker, il ne s'agit pas de concevoir les formes grammaticales comme les reflets d'une réalité objective mais comme les reflets de notre perception du monde. Il convient donc d'entendre «espace » non pas au sens de l'espace physique qui nous entoure mais de l'espace tel que nous le vivons (le vécu expérientiel). Autrement dit, Il ne s'agit plus de l'espace «brut» mais d'un espace " anthropologisé ». Les formes linguistiques, selon cette conception, "incarnent» ( embody) l'interaction de l'homme avec son environnement. Le fait d'orienter le signifié vers l'expression de relations et non d'objets constitue selon nous un progrès important par rapport au localisme naif. Il reste que ces relations demeurent appuyées sur des données spatiales de type référentiel externes au langage.

Pour B. Victorri $(1999,2010)$, les prépositions ont pour fonction de construire des « scènes verbales » dont, dit-il, la topologie est « très loin de s'identifier à la topologie de l'espace physique » (1999: 99), et les descriptions purement physicalistes sont selon lui « vouées à 
l'échec car elles se centrent sur la référence, sans tenir compte de la liberté qu'offre la parole de construire des espaces anthropologisés conçus en fonction des rapports qu'on entretient avec le monde » $(1999$ : 100). Cette liberté que souligne pertinemment Victorri, comprise dans le sens de l'autonomie du système, est une thèse capitale. Mais, dans l'interaction de l'homme avec son environnement, Victorri entend maintenir le principe d'un sens concret, ancré dans les expériences corporelles. Dans ces conditions, le passage aux sens non spatiaux et conceptuels devra être expliqué par des "phénomènes de transfert conceptuel entre domaines» (Lapaire 2017). L'élément explicateur principal reste alors la métaphorisation comme autrefois le «sens figuré ». Le référentialisme direct des thèses "physicalistes » est intenable en linguistique, comme le dit Victorri, mais les notions de "topologie ", «localisation» ou "repérage " telles que les utilise l'auteur trahissent finalement un référentialisme externe indirect. Il s'agit de localisations dans un espace autre que physique, certes, mais qui est défini comme une image de l'espace physique. Ce qui change alors c'est seulement, pourrait-on dire, le degré d'investissement de l'organisme dans l'espace.

On peut se demander pourquoi, au risque du référentialisme, les grammairiens se tournent davantage vers les relations spatiales pour expliquer les phénomènes linguistiques, plutôt que vers le fonctionnement auto-entretenu du système linguistique en tant que tel. Une approche centrée sur le système devrait en effet essayer d'expliquer comment ce système émerge et se maintient dans un milieu complexe et a priori peu ou non favorable. La théorie générale des systèmes pose que cela se produit à partir des ressources propres du système. Les déterminations externes sont inévitables, mais si elles sont actives, on sait que ce ne sera pas dans le sens du maintien du système. Au contraire, ces déterminations œuvreront pratiquement toutes (sauf exception fortuite) dans le sens de l'entropie, et finalement du désordre et de la déstructuration. La condition même du maintien du système, voire de sa survie, repose alors précisément sur son autonomie et son auto-organisation. Le préfixe « auto » n'est pas ici décoratif. C'est le point central de la théorie. La théorie des systèmes appliquée au langage aboutit donc obligatoirement à l'auto-référence, à l'opposé de toute primauté ou primarité de causalités externes ${ }^{21}$. Pour elle, le système se constitue en liaison avec le monde, mais ce n'est pas dans le monde qu'il faut chercher la clé de son organisation interne ou même des relations externes qu'il entretient avec lui. La métaphorisation (et / ou métonymisation) pose problème car elle implique toujours un ancrage initial déterminant dans ce qui est métaphoriséz ${ }^{2}$.

31 Il est incontestable que le langage entretient avec l'espace (mais aussi le temps) des relations multiples, étant donné que nous vivons dedans. Mais la primarité / primauté de l'espace ne peut être qu'un postulat: ce n'est pas parce qu'on trouvera dans toutes les langues de nombreuses métaphores spatiales que l'espace est primaire dans la construction du langage. La métaphorisation, dans le plan communicationnel, présente des avantages considérables en faisant appel à des expériences basiques, imagées, immédiatement partagées a priori. Il ne s'agit donc pas de nier son rôle, qui est capital, mais de ne pas en faire la cause première du système. La thèse d'un ancrage de l'abstrait dans la concrétude spatiale n'explique d'ailleurs absolument pas comment des sens abstraits peuvent émerger et se développer. Il faut au minimum qu'il y ait dans le langage un moteur puissant de l'abstraction, ou à tout le moins un déterminisme fort dans cette direction. L'abstraction par rapport à l'expérience concrète, la mise en perspective des différents contextes, l'établissement de relations inter-organismes, sont précisément les prises de distance que le système auto-organisé du langage permet d'opérer. On peut 
affirmer que c'est l'abstraction en quelque sorte "native" du langage qui permet le développement cognitif. Les thèses spatialistes, même non strictement physicalistes, paraissent prendre ainsi le problème totalement à l'envers.

Il faut selon nous concevoir le système langagier comme un moteur de l'abstraction et expliquer comment, en généralisant l'expérience individuelle, il rend possible le phénomène de l'échange, du partage interlocutif. Or ce partage est généralement mentionné comme une conduite ordinaire et naturelle. Il est vu comme un donné alors qu'en réalité, c'est précisément la grande question à résoudre : comment un partage cognitif autre que très rudimentaire peut-il s'établir entre des expériences privées? Il faut un travail considérable pour établir ce partage et des relations qui ne se contentent pas de dire l'état des choses. C'est pour cette raison que le langage est un système. Nous devons donc nous intéresser aux relations véritablement primaires, fondatrices, celles qui vont permettre à l'organisme de s'abstraire de la concrétude et de se "dé-subjectiviser » pourrait-on dire, afin de trouver un système de partage et d'accord avec les autres organismes. Il ne peut trouver de terrain d'entente avec ses partenaires communicationnels que grâce au système linguistique qui, très en profondeur, construit et stabilise les conditions et les modalités de cette dé-subjectivisation. Présupposer des « connaissances partagées » (shared knowledge), c'est tout simplement mettre sous le tapis toute la problématique constructionnelle du langage.

La subjectification / intersubjectification de Traugott \& Dasher (2002) essaie d'apporter une réponse en introduisant la question de l'interprétation des signes et des messages. C'est en interprétant qu'on serait naturellement amené à faire dériver, si peu que ce soit, le message d'origine, ce qui amorcerait le changement sémantique en diachronie. Pour son étude de UP, F. Toupin (2006) se réfère à ce cadre de la pragmatique historique :

Les lexèmes suivent universellement une évolution sémantique en trois étapes, qui les conduit de l'expression de la spatialité (expression des relations spatiales) à celle $\mathrm{du}$ point de vue du locuteur (ang. Subjectification - point de vue étant à prendre au sens large, et non par exemple au sens restreint d'une modalité appréciative) et enfin à l'encodage de l'attitude du locuteur vis-à-vis de son interlocuteur dans le « ici et maintenant » de l'acte de parole (ang. intersubjectification»). (F. Toupin 2006 : §3)

Selon son analyse, la complexité sémantique de UP s'expliquerait par la cohabitation en anglais contemporain de ces modules, «le premier module historique, celui qui permet l'expression des relations spatiales, n'ayant pas disparu avec l'émergence des autres modules» (2006: \$42). L'auteure ajoute que ces modules forment «un continuum qui mène, dans un ordre qui est sans doute un universel, du spatial à l'intersubjectif en passant par l'expression du point de vue de l'énonciateur» (2006: \$42) (c'est nous qui soulignons). Ce modèle apporte un élément d'explication plus solide que les modèles ordinaires car, selon sa logique, c'est l'échange et l'intersubjectivisation qui provoquent la métaphore et l'abstraction. Mais le principe cognitiviste demeurerait à l'amorce de l'évolution : le système linguistique traduirait d'abord l'expérience individuelle concrète et serait amené seulement après, dans l'histoire, à se grammaticaliser (se métaphoriser) de plus en plus. Outre qu'on fait appel en ce cas à l'accident historique plutôt qu'à un véritable "moteur » systémique, il faut aussi reconnaître qu'il serait très curieux que la métaphore qui est justement basée sur un appel au concret pour mieux assurer l'intercompréhension, soit précisément le moteur de l'abstraction et de l'éloignement de la concrétude ${ }^{23}$. 

considérer comme données de départ deux organismes en collaboration, interlocutivement reliés (par le même système linguistique oral). Si ces deux interlocuteurs se trouvent dans le même «contexte " physique, chacun d'eux en a sa propre perception, du simple fait que chaque sujet devient à son tour objet de la perception de l'autre: les visions sont toujours dissymétriques. Il y a donc nécessairement non pas un, mais deux contextes à considérer, celui du locuteur et celui de son interlocuteur. Ces deux contextes distincts se présentent à l'état d'«univers mentaux ", qui sont a priori non mutuellement accessibles directement. Comme l'écrit A. $\mathrm{H}$. Gardiner à la suite de la citation en exergue de cet article: "Sympathy and understanding are indeed possible, but two minds cannot interpenetrate one another in any literal sense ${ }^{24} »(1932: 67)$ [L'empathie et la compréhension sont certes possibles mais deux esprits ne peuvent s'interpénétrer l'un l'autre ${ }^{25}$ au sens propre]. Si, par exemple, deux personnes voient ensemble un même coucher de soleil, leurs deux expériences seront incommensurables. Le langage s'établit précisément sur cette différence, cette fracture originelle : c'est sa raison d'être de permettre l'échange sous ses deux aspects, comme une activité sociale d'information et de communication en «aval» des organismes, mais aussi et surtout en "amont» de leur constitution profonde comme la seule manière possible pour le système de s'unifier et de s'auto-organiser.

Notre préambule est donc qu'il est impossible de partir de l'idée qu'il pourrait y avoir d'emblée une perception commune, partagée, des contextes. Le contexte, comme le définit fort bien P. Schmoll (1996: 243), c'est ce qu'il y a dans la «tête» de chacun des interlocuteurs et qui est donc inaccessible $a$ priori pour autrui :

Le contexte pertinent est [donc] à la fois ce qu'il y a de plus stable dans le contexte, ce qui est toujours là, dans la mémoire, même si les données objectives varient, mais comme c'est ce qu'il y a dans la tête des locuteurs, c'est en même temps ce qui est le plus inaccessible.

Comment sortir de ce contexte privé, comment réussir ce que F. Jacques appelle cet « improbable accord de deux totalités privées » $\left(1979: 48^{26}\right)$ ? Notre hypothèse est que le moyen de parvenir à l'accord interlocutif est de construire, au moyen du langage, un contexte commun, une co-contextualisation, qui permette de comprendre les signes au sens de "prendre en même temps, s'entendre ». La clé du fonctionnement du langage réside précisément là. À rebours des conceptions pour qui les formes langagières reflètent, voire incarnent, la perception subjective que nous avons du monde, nous pensons que le langage offre au contraire à l'homme la possibilité de sortir de sa subjectivité, de son univers "privé »- condition nécessaire à la possibilité même de la communication.

Nous considérerons en conséquence qu'il n'y a aucune raison pour laquelle une quelconque concrétude spatiale s'imposerait au départ. Ce ne sont pas des relations spatiales ou des repérages qui sont à la racine de UP ou d'autres particules / prépositions en anglais, même si ces particules permettent dans des contextes appropriés de signifier des relations spatiales et de se repérer. Les valeurs de UP généralement invoquées dans le 
modèle spatial trouvent leur explication systémique profonde dans le besoin du système de se mobiliser et de se répliquer sans cesse, pour son auto-entretien (self maintenance). La logique qui informe le langage n'est pas une logique spatiale mais une logique communicationnelle.

\section{Configurations systémiques} (Douay \& Roulland 2014), nous avons montré que le système du langage était un système communicationnel et devait être décrit comme tel. Un organisme isolé, même considéré de manière naturalisée dans sa relation à son environnement, ne peut pas élaborer un tel système. Il lui faudrait une intention et des capacités de conscience que précisément le langage ne lui pas encore données. Ce serait mettre la charrue avant les bœufs. Il lui faut l'appui d'un terme distinct de lui afin d'opérer une triangulation qui lui permette d'objectiver ses contenus de pensée et ses perceptions. Ce terme peut être obtenu si cet organisme est en mesure de comparer son expérience à un organisme similaire associé. C'est pour cette raison que, dans notre modèle interlocutif, les parties premières du langage, les données offertes à l'observation du linguiste, ne sont pas les signes linguistiques déjà construits et opérationnels (morphèmes, lexèmes, constructions, etc). Ce sont avant toute chose les deux interlocuteurs - les deux organismes en relation - qui communiquent entre eux, échangent et comparent leurs expériences respectives en se référant au terme objectif commun qu'est le système linguistique. Ce qui importe dès lors, c'est ce terme objectif, le système, qui coordonne les expériences à la fois différentes et identiques des interlocuteurs.

Dans le cadre de la théorie générale des systèmes, nous avons décrit le le système linguistique comme une relation dynamique entre les interlocuteurs, ce dynamisme produisant et reproduisant incessamment par réplication les signes « objectifs ». C'est la réplication qui, in fine, rend compte à la fois de la structure linguistique et de son usage pragmatique. Il n'est pas possible de développer ces aspects ici, et nous renvoyons le lecteur à Douay \& Roulland 2014. Pour cette étude sur UP, nous allons simplement rappeler les caractéristiques principales des trois termes de la triangulation.

Comme nous l'avons souligné supra, pour que l'expérience soit partagée ou tout au moins mise en situation de partage, un système ne peut pas s'établir sur un seul organisme. A l'action d'un "émetteur» de message (que nous appelons $\alpha$ ) correspond toujours une action de validation et d'interprétation de la part d'un organisme "récepteur " complémentaire (que nous appelons $\beta^{27}$ ). Nous appelons notre théorie "interlocutive " car aucun système linguistique ne peut s'établir en dehors de l'échange communicationnel, même si, au niveau systémique, les schémas communicationnels sont des formes abstraites avant d'être des échanges concrets. Ces schémas se stabilisent et s'articulent comme des états de système. Nous appelons ces états des «configurations ». Selon la triangulation, nous devons obtenir basiquement deux configurations interlocutives opposées ( $\mathrm{C} 1$ et $\mathrm{C} 2$ ) et une configuration centrale résolutive ( $\mathrm{C} \varnothing \mathrm{ou}$, comme nous l'appelons généralement, RID pour «Rapport Interlocutif Direct»). Une configuration peut être définie comme un «cadre réceptif " puisque rien ne vaut qui ne soit reçu. Les cadres réceptifs se construisent en opposition selon que le centrage a lieu sur l'émetteur $\alpha$ ou le récepteur $\beta$. Pour UP et DOWN, nous avons une opposition de ce type, avec un centrage $\alpha$ pour UP et un centrage $\beta$ pour DOWN. 

rapport à laquelle les deux autres $\mathrm{C} 1$ et $\mathrm{C} 2$ se déterminent.

\section{Configuration CØ ou RID}

44

Cette configuration est celle du signe lui-même, avec toute son autonomie et son indépendance en tant que forme de langue, par rapport aux valeurs contextuelles $\alpha$ et $\beta$. C'est en relation avec cette autonomie et cette objectivité ${ }^{28}$ qu' $\alpha$ et $\beta$ positionnent leurs propres caractéristiques. On peut définir cet état comme un état d'équilibre qui correspond à ce qu'on peut appeler la «norme » dans la mesure où elle n'est interprétée contextuellement ni par $\alpha$ ni par $\beta$. La norme linguistique est donc dans notre modèle le produit du partage interlocutif, de la construction systémique de ce partage. Cette construction aboutit à une sorte de "pacte interlocutif» qui, du fait même de sa construction, est à la fois une convention et une réalité objective. Les formes de RID d'une langue donnée sont des formes « objectives " au sens où elles sont tenues pour valables à égalité par tous les interlocuteurs de cette langue, quelle que soit la variation individuelle. Elles renvoient au sens « commun ${ }^{29}$ » ou réputé tel. Ce faisant, mais étant relatives à ces variations, elles les autorisent et, pour cette raison, l'inférence joue à plein pour l'interprétation discursive.

Il faut faire très attention à cette question de "norme». Le mot a tendance à être immédiatement compris au sens d'une norme sociale. Or la norme linguistique ne doit surtout pas être comprise ainsi, du moins pas à ce niveau de construction systémique. Les parties en cause à ce stade ne sont pas des êtres sociaux constitués et empiriques. Ce sont des rôles systémiques, ou, si l'on veut, des places relationnelles. En ce sens, la norme linguistique est à cette étape non instanciée, et ne concerne qu'une convention de principe entre les deux participants $\alpha$ et $\beta$. Il en résulte que la norme linguistique telle que le langage peut la livrer doit être constamment interprétée. C'est pour cette raison que les formes de RID exigent un contexte pour être comprises.

Un exemple simple pourra fixer les idées : le nom propre sans article en français ou en anglais ( $\varnothing$ Marie, $\varnothing$ John, etc.) renvoie à la personne qui lui est "normalement", «communément» attachée, en vertu du «pacte linguistique» conclu par deux interlocuteurs qui engagent une relation interlocutive. Il n'y a rien d'autre à y voir. C'est pareil pour les noms communs, à ceci près que l'organisation grammaticale générale des différentes langues interfère nécessairement et engendre des fonctionnements qui peuvent diverger d'une langue à une autre. $\varnothing$ Water en anglais peut par exemple renvoyer à de l'eau en général ou désigner une eau particulière, ce qui n'est pas le cas du français $\varnothing$ eau. $^{30}$ signe «signifie » quelque chose, mais qu'il soit employé et surtout réemployé par des interlocuteurs dans des situations qui sont toutes nécessairement différentes. C'est ce réemploi qui le fait signifier. Pour cette raison, la clé du fonctionnement du système est la réplication, qui consiste dynamiquement à renouveler le «pacte linguistique » dont relève le signe.

$\mathrm{Au}$ lieu de prendre la voie centrale et égalitaire du RID, la réplication peut adopter le cadre réceptif de l'émetteur $\alpha$ ou bien celui du récepteur $\beta$, dans les configurations $\mathrm{C} 1$ et 
C2. Nous allons d'abord examiner la configuration C1, qui nous importe particulièrement ici car c'est celle dont relève UP.

\section{Configuration $\mathrm{C} 1$}

directement à lui-même et, par conséquent, remet en cause le pacte dont il est théoriquement porteur. Faire valoir cette configuration pour un signe donné, c'est faire dire au signe autre chose que ce qu'il dit "normalement » quand il est censé renvoyer à un sens réputé d'emblée commun. De la sorte, l'émetteur se réapproprie le signe et «singularise » son expérience personnelle face à d'autres expériences potentielles. La promotion de cette nouvelle interprétation du signe consiste donc très clairement en une rupture ou « disruption " par rapport à un état d'équilibre théorique. Le locuteur réel qui utilise une forme $\mathrm{C} 1$ marque à l'endroit de son interlocuteur une manière ou une autre de singularité et de différence par rapport à ce que cet interlocuteur est censé avoir à l'esprit.

Cette disruption peut se décliner de multiples manières. Il peut s'agir de marquer une action, une mobilisation, une initiative, de la part du locuteur qui signale une différence eu égard à une norme (impliquée). Du fait de la disruption par rapport à la situation d'équilibre, le signe se trouve décalé d'une norme en ce qu'il revendique une action originale, norme qui, nous le rappelons, est indéterminée et ne se confond pas avec une quelconque norme sociale. Mais ce faisant, il revendique aussi une contextualisation qui n'est plus réputée commune. La configuration $\mathrm{C} 1$ est par essence différentielle et surdistinctive. A cet acte de sur-distinction ${ }^{31}$ sont liées les valeurs qu'on peut globalement qualifier de «hors-norme». UP, comme nous le verrons, apporte toujours à la construction cette dimension d'exceptionnalité.

51 En français ou en anglais ${ }^{32}$, par exemple, l'article indéfini peut accompagner le nom propre : il donne alors à voir une image décalée, inédite ou « hors-norme », du référent normalement désigné (par exemple: une Marie bien fatiguée est apparue hier ${ }^{33}$ ). Le nom implique par définition le partage interlocutif, mais l'article de son côté marque une réplication du pacte en rupture avec un équilibre supposé ou de principe. Toutes les formes $\mathrm{C} 1$ se caractérisent ainsi par des valeurs fortement sélectives et différentielles. Le terme «acte de disruption ${ }^{34}$ " permet de souligner qu'il s'agit non pas d'un aléa, d'une perturbation accidentelle venant nuire au bon fonctionnement du système mais d'un acte interlocutif au sens plein et dynamique du terme, un acte généralement discursivement fort qui marque une forme ou une autre de saillance ou de différence. Il est très important de bien comprendre que le détail de l'interprétation sera intégralement dû à l'inférence contextuelle.

Il y a fondamentalement dans C1 l'expression d'un dédoublement intérieur, lequel s'explique par le retour pratiqué sur le pacte interlocutif. Ceci interdit de positionner une limite externe ou globale quelconque. Le périmètre de signification de l'expression, non clos, apparaît non conclusif. De là les valeurs d'indétermination qui s'attachent aux formes $\mathrm{C} 1$, ce qu'illustre l'article indéfini ci-dessus. On pourrait les qualifier de valeurs « imperfectivantes » étant donné que l'expression laisse ouvert un potentiel interlocutif souvent important.

On aura compris que ce qui est en cause pour le système et sa construction n'est pas le défilé des objets du monde dont pourrait se saisir l'observateur. Ce qui est en cause, c'est 
l'observation elle-même, l'angle de saisie que l'observateur adopte lors de la construction de son message.

\section{Configuration C2}

54 La configuration C2 est centrée sur le récepteur $\beta$. Il n'y a donc pas là de retour analytique sur un pacte mais au contraire confirmation et pérennisation de ce pacte. De ce fait le pacte interlocutif est doublement ratifié. L'expérience individuelle, dans ce cadre réceptif, n'a aucune liberté de variation. Elle est déclarée impérativement conforme au message émis et ne peut en diverger en aucune manière. Deuxièmement, cette conformité déborde nécessairement toute la contextualisation présente. Il en résulte que, là où $\mathrm{C} 1$ apparaît essentiellement différentielle, $\mathrm{C} 2$ fonctionne sur l'identité et sa pérennisation : l"objet saisi est toujours le même quel que soit l'angle de saisie. Ainsi la différence est liée à l'intériorité en $\mathrm{C} 1$ : la différence joue entre des parties internes. Au contraire en C2 l'identité est liée à l'extériorité.

Si la nouveauté, l'originalité, le hors-norme, la déviance (avec tous les effets afférents), sont liés à l'acte de sur-distinction marqué par $\mathrm{C} 1$, c'est au contraire l'attendu, le prévisible, la conformité aux attentes, la « ligne droite », l'uniformisation, liés au refus de la divergence, qui sont marqués par C2. En français, l'article défini est une marque C2. Avec le nom propre, il met en avant le côté figé et immuable, par exemple, d'un comportement. C'est ce qu'on aurait dans La Marie, de toute façon, elle n'en fera qu'à sa tête $e^{35}$. Entre Ø Marie, une Marie et la Marie, il n'y a pas trois « sens » différents, mais une même réplication qui prend trois voies interlocutives différentes, qui correspondent aux trois configurations de notre modèle.

Le tableau ci-dessous récapitule de façon schématique, simplement pour fixer les idées, les caractéristiques respectives des trois configurations et les principaux effets de sens afférents :

\begin{tabular}{|c|c|c|}
\hline $\begin{array}{ll}\text { CØ ou } \\
\text { RID }\end{array}$ & $\begin{array}{l}\text { ACCORD } \\
\text { CONVENTION / NORME }\end{array}$ & $\begin{array}{l}\text { - Renvoi au sens « commun » partagé } \\
\text { - Norme interlocutive de principe } \\
\text { - Etat d'équilibre }\end{array}$ \\
\hline $\mathrm{C} 1$ & $\begin{array}{l}\text { DISCORDANCE } \\
\text { DISRUPTION / } \\
\text { DIFFERENCE }\end{array}$ & $\begin{array}{l}\text { - Discontinuité, décalage, divergence interlocutive } \\
\text { - Contestation de la «norme» } \\
\text { - Fragmentation, dédoublement } \\
\text { - Acte de sur-distinction, valeurs fortement sélectives } \\
\text { - Valorisation de l'observation, du sujet observant, } \\
\text { perspective analytique } \\
\text { - Sur-mobilisation } \\
\text { - Expression de l'inattendu } \\
\text { - Valeurs non conclusives («imperfectivantes ») }\end{array}$ \\
\hline
\end{tabular}




\begin{tabular}{|c|c|c|}
\hline $\mathrm{C} 2$ & $\begin{array}{l}\text { CONCORDANCE } \\
\text { CONFORMITE / } \\
\text { IDENTITE }\end{array}$ & $\begin{array}{l}\text { - Continuité, convergence interlocutive } \\
\text { - Conformité avec la «norme » } \\
\text { - Globalité, unification } \\
\text { - Acte de sous-distinction, valeurs anti-sélectives, } \\
\text { nivellement } \\
\text { - Valorisation de l'objet observé } \\
\text { - Perspective synthétique, uniformisation } \\
\text { - Nécessité et prévisibilité } \\
\text { - Valeurs conclusives («perfectivantes») }\end{array}$ \\
\hline
\end{tabular}

\section{Un sens unique pour les emplois de UP}

En ce qui concerne l'écueil consistant à « surinvestir le mot pour ne rien rater de sa spécificité », il est évitable à condition de commencer l'étude linguistique par une interrogation sur le processus interprétatif au terme duquel le mot prend littéralement sens, «fait» sens. La valeur de base d'une forme linguistique, quelle qu'elle soit, configure un "cadre réceptif », déterminant pour l'interprétation, mais elle interagit au niveau discursif avec des facteurs co(n)textuels (modalités prosodiques et kinésiques, contexte ou situation au sens large) et c'est en prenant simultanément en compte tous ces facteurs que l'interlocuteur infère le sens véhiculé par les différents signes et in fine la pertinence de l'énoncé qui lui est adressé. Il n'y a pas de sens en soi en dehors de cette interprétation. Faute de prise en compte de ces facteurs et de la stratégie inférentielle requise pour l'acte d'interprétation, on arrive en effet à vouloir faire dire à la forme plus que ce qu'elle dit et plus que ce qu'elle a à dire. On risque par ailleurs de confondre la valeur systémique ${ }^{36}$ de la forme considérée et la valeur pragmatique de l'énoncé dans lequel elle est utilisée, autrement dit d'attribuer à la forme un sens que, sans s'en rendre compte, le linguiste infère de ces autres facteurs. Comme l'expliquait Gardiner (1932), toute théorie grammaticale doit s'adosser à une théorie de l'interprétation explicitement formulée en préambule (Douay 2000 : 49-53). Par conséquent, nous pouvons considérer que la diversité des interprétations de UP est la résultante de l'interaction entre sa valeur systémique, unique, invariante, qui détermine un «cadre réceptif » spécifique, et la variété des co(n)textes où elle se trouve utilisée.

Quant à la difficulté évoquée par Franckel \& Paillard à propos du niveau d'abstraction de l'« épure » qui risque de rendre ardu le raccord entre invariant et effets de sens, elle découle de l'idée très répandue que les valeurs systémiques sont toujours peu ou prou, comme nous l'avons expliqué, ancrées dans le réel physique ou le réel vécu. Valeurs qu'on ne peut ensuite raccrocher aux valeurs communicatives observables dans l'usage qu'à l'aide de processus de dérivation métaphorique. A. Culioli de son côté fait intervenir des 
concepts tel que celui de «déformabilité ». On peut aussi supposer des règles complexes permettant de rendre aptes à la communication des formes qui, au départ, n'auraient pas vocation à communiquer ${ }^{37}$. J.M. Moeschler $(2001: 100)$ se risque ainsi à dire que la communication n'est pas la fonction première du langage.

La conception que nous défendons de valeurs systémiques de nature exclusivement relationnelle permet d'emblée de régler ce problème du fossé entre invariant et usage : le système produit des formes en vertu de propriétés qui sont de nature communicationnelle et non en vertu de propriétés autres du monde ou de l'esprit. La communication n'est pas seulement une fonction du langage, elle en est la forme même. On ne répètera jamais assez l'aphorisme saussurien que « la langue est une forme et non une substance » (Saussure 1973 : 169). C'est au prix de l'auto-référence que la valeur d'une forme peut être qualifiée d'invariante, l'auto-référence du système qui lui permet de construire ses relations avec l'environnement. Ce n'est pas l'environnement qui impose au système les relations en question et s'il s'agit d'une interprétation de l'environnement, alors il faut expliquer comment l'organisme établit cette interprétation.

61 L'étude discursive ${ }^{38}$ qui suit a donc pour objet de montrer qu'il est possible de lire dans UP une valeur relationnelle unique et invariante, quelle que soit la base affectée ou la relation mise en cause. En aucun cas UP ne présuppose un cadrage spatial ou topologique qui serait déterminant. Au contraire, ce cadrage, qui semble si immédiat, est en réalité une construction sur la base des propriétés de UP comme marque interlocutive C1. Il n'y a rien au niveau systémique qui signifie "vers le haut » mais les caractéristiques de C1 dont relève UP le rendent apte à évoquer l'émergence ou la saillance, qui pourront à la faveur de certains contextes où prédomine l'interprétation spatiale ou physique, être interprétées dans le sens d'un mouvement vertical vers le haut. Fondamentalement, cette verticalité et cette direction vers le haut ont comme soubassement un mouvement systémique de nature interlocutive. Nous avons vu supra que la configuration $\mathrm{C} 1$ consiste en une disruption par rapport à une "norme ». Cette norme est celle d'un accord équilibré, sans aucune saillance ou émergence disruptives. "Vers le haut » signifie en réalité « à contre-sens » de la norme ou en rupture avec elle.

Il en est de même pour DOWN, marque interlocutive C2 qui répond à UP. Cette particule peut s'appliquer à un mouvement orienté vers le bas mais ce n'est pas là son invariant. En liaison avec UP mais à l'inverse, DOWN implique une conformité absolue à la "norme " que conteste UP. Elle s'applique en effet à des situations où sont valorisés la continuité, le cours inexorable des choses, la nécessité, voire l'extinction, à l'opposé absolu de l'émergence. Dans un cadre réplicationnel, celui de tout acte de langage, DOWN marque donc la prédominance de la norme auquel tout acte doit référer. C'est là l'invariant de la configuration $\mathrm{C} 2$ dont il relève.

63 Il nous paraîtrait extrêmement difficile et compliqué d'obtenir par des processus de dérivation métaphorique ou métonymique les valeurs systémiques de disruption vs conformité à partir de représentations spatiales du type mouvement vers le haut ou vers le bas. En revanche, il est très facile d'expliquer les valeurs spatiales à partir des configurations interlocutives $\mathrm{C} 1$ et $\mathrm{C} 2$ comme bases systémiques de structuration. La disruption de $\mathrm{C} 1$ marque en effet une forte mobilisation du sujet en opposition à la tendance normative. Si tant est qu'il faille structurer mentalement des mouvements spatiaux, le système opposera un mouvement actif mobilisant et saillant à un mouvement inverse. Le système offre ainsi élégamment aux repérages spatiaux sa propre binarité 
relationnelle. Sans les relations réplicationnelles du système, l'organisme n'aurait aucun moyen de structurer linguistiquement l'espace.

L'analyse des exemples que nous examinons ci-dessous a pour but de relier entre elles les interprétations attestées de la particule UP dans leur variété et aussi d'expliquer ce qui permet ou pourrait permettre son interprétation correcte dans les cas où elle est n'est que susceptible d'apparaître. Nous allons essayer de présenter les usages contextuels de UP de la manière la plus ordonnée possible.

En premier lieu, UP, dont l'invariant relationnel, dans le cadre de la TRI, se définit comme marque interlocutive de type $\mathrm{C} 1$, se prête à l'expression de tout ce qui est donné à voir comme hors-norme. La déviance par rapport à un cours attendu des choses apparaît très nettement dans tous les cas où quelque chose ne se passe pas normalement ou pourrait ne pas se passer normalement. Ces cas sont les plus courants et sans doute serait-il souhaitable de les voir figurer en premier dans les dictionnaires. La traduction française exprime parfois très clairement cette idée d'inattendu dans, par exemple :

4. Unless something comes up « à moins d'un imprévu »

Come sans UP ne traduirait pas la notion d'émergence ou de survenue inattendue et importante (de nature à modifier le cours des choses). Autres exemples, avec des valeurs très proches :

5. What's up? « Qu'est-ce qui se passe?» $\mathrm{Ou}:$

6. What are you two up to? «Qu'est-ce que vous mijotez tous les deux? ?

68 C'est la question couramment posée quand il y a perception de quelque chose de relativement anormal. Le dictionnaire en ligne Cambridge Dictionary, après n'avoir donné que des exemples où UP, selon son interprétation, exprime un mouvement vers le haut ("toward or in a higher position») note toutefois que, dans le langage familier (« informal»), UP peut avoir le sens de "wrong»: «We say that something is up when something is wrong »). C'est visible dans l'exemple suivant:

7. Katrina was in that morning. She noticed that something was up and she came over to see me. 'What's wrong', she asked. 'Nothing, nothing's wrong. I've had rather a surprising piece of news, that's all'. 'Why, what's up'? "Katrina était sur place ce matin-là. Elle a remarqué qu'il y avait quelque chose d'anormal / que quelque chose n'allait pas, et elle est venue me voir. 'Y a un problème ?' m'a-t-elle demandé. 'Non, non, y a rien, tout va bien. J'ai juste reçu une nouvelle surprenante, c'est tout'. 'Et alors? Qu'est-ce qui se passe ?'«

Cet effet de sens que le dictionnaire constate et n'explique pas est imputable à la disruption par rapport à la norme, caractéristique de la configuration C1 dont relève UP. L'interprétation dans un sens négatif reste à calculer selon les contextes par rapport à la normalité que les interlocuteurs attendent ou présupposent. UP évoquant de par sa valeur systémique l'émergence d'une situation disruptive, il peut aussi s'agir simplement de demander à l'interlocuteur s'il y a du nouveau dans sa vie («Quoi de neuf?»). L'écart par rapport au «normal» est toujours tributaire de l'appréciation des interlocuteurs dans la situation locale.

La disruption par rapport au cours normal des choses transparaît aussi très nettement dans les exemples que nous avons cités en introduction : play up (The dishwasher is playing up again "Le lave-vaisselle fait encore des siennes») ou mess up (Somebody has messed everything up again "Quelqu'un a encore tout fichu par terre / en l'air »). De même le verbe act up s'emploiera pour signifier un comportement déviant (" faire l'imbécile ») : 
8. Children may act up in class in an effort to get attention (Wiktionary) « Il arrive que

les enfants fassent l'imbécile en classe juste pour attirer l'attention »

ou un mauvais fonctionnement :

9. Our car always acts up in bad weather " Notre voiture tombe toujours en panne quand il fait mauvais temps »

De façon générale, on peut s'attendre à trouver UP dans tous les cas où, pour le dire familièrement, quelque chose est perçu comme allant de travers (voir botch up "bousiller», screw up "se planter») ou quand il y a un raté (pass up an opportunity «manquer une occasion»). De même slip up, employé comme verbe ou nom (a slip-up), renvoie, au sens propre comme au sens figuré, à un dérapage, un incident imprévu, une erreur de parcours (exemples 10 et 11 extraits de Linguee) :

10. There will be a high price to pay for the slightest slip-up at the moment of truth - the finishing line. "Le moindre faux pas coûtera très cher à l'heure des bilans » (traduction de Linguee)

11. Employees who are used to emphasizing a certain side to their personality, and even showing different sides to different people, may slip up occasionally. "Les salariés qui ont l'habitude de mettre l'accent sur une facette précise de leur personnalité, et même de montrer des visages différents selon les personnes, peuvent occasionnellement commettre des impairs ». (traduction de Linguee)

12. If you slip up, don't give up «En cas de rechute, ne baissez pas les bras ${ }^{39}$ (formule souvent utilisée par les psychologues)

Il est intéressant et très important de noter cette tendance à l'interprétation défavorable. UP est la marque d'une perturbation de la norme, ce qui s'accompagne d'un calcul axiologique variable mais volontiers négatif. Baby up, cité en (3) (« infantiliser »), évoque défavorablement un comportement jugé hors-norme (« traiter un adulte en bébé »). Mais le degré effectif d'anormalité et de non conformité aux attentes exprimé par UP est bien entendu toujours fonction du contexte :

13. I don't like people turning up without an appointment «Je n'aime pas les gens qui débarquent sans avoir pris rendez-vous "

14. Is this your pencil that turned up in my drawer? « C'est ton crayon qui a atterri dans mon tiroir?

On peut s'étonner, au vu de ces interprétations très courantes de UP autour de l'idée de disruption (ce qui explique que, dans la plupart des exemples que nous avons relevés, il s'agit de contextes où advient quelque chose de non souhaité), que de nombreux linguistes, à la suite de Lakoff et Johnson persistent à associer UP à l'idée d'un mieux ( better). La raison qu'ils donnent eux-mêmes est la priorité qu'ils attribuent au sens spatial :

More is better is coherent with more is up (Lakoff \& Johnson $2003: 23$ )

Le jugement qu'on peut porter en termes positifs ou négatifs est affaire d'inférence contextuelle. Ce sont uniquement les facteurs co(n)textuels qui permettent d'inférer la nature et la force exacte de l'exception à la norme.

Ceci dit, le jugement peut se faire aussi neutre que possible. Dress up, par exemple, signifie fondamentalement «ne pas être habillé comme à l'ordinaire, s'habiller (expressément) de manière distincte ». Seul le contexte permet d'inférer si la personne est « sur son trenteet-un ", dans un sens laudatif, ou "endimanchée », dans un sens péjoratif, ou encore "déguisée " (au sens propre ou figuré) ou tout simplement, comme dans l'exemple suivant (15), vêtue d'habits qui ne sont pas normalement les siens ${ }^{40}$ : 
15. [l'inspecteur Morse s'oriente vers l'hypothèse que Mrs Taylor est sortie vêtue de l'uniforme porté par sa fille quand elle se rend au lycée pour faire croire que celleci n'était pas morte]

Let us assume that Mrs Taylor dresses up as her daughter and makes her way towards school «Supposons que Mrs Taylor mette les vêtements de sa fille et se dirige vers

l'école » (Colin Dexter, Last seen wearing, p. 225)

Dans les exemples ci-dessous, en collocation familière avec a storm, le degré du horsnorme est hyperboliquement renforcé :

16. We ate up ${ }^{41}$ a storm ! « on a mangé / bouffé comme des malades!»

17. She cooks up a storm! «C'est une cuisinière hors pair / exceptionnelle !»

18. When I'm in London, I shop up a storm "Quand je suis à Londres, je fais une grosse

cure de shopping"

Cette notion de hors-norme véhiculée par UP est rendue lexicalement en français, faute d'un système de particules verbales. Dans nos traductions des phrasal verbs cités en exemple, nous avons eu recours à un verbe lexical spécifique ou une expression dite idiomatique (« mijoter, faire des siennes, ne pas tourner rond, faire l'imbécile, débarquer, atterrir ", etc.). Comme nous l'avons vu, en anglais, la particule est vectrice d'une part considérable du signifié de la construction. Les verbes auxquels est associé UP ont généralement un sens vague, plus modal qu'actionnel (put, set, stand, act, turn, etc.). Contrairement à l'opinion (répandue) de Denison, qui considère que la particule des phrasal verbs complète adverbialement le verbe lexical, nous observons que c'est UP qui est déterminant pour l'interprétation, et non l'inverse. Le verbe lexical apparaît davantage comme complémentaire, ou, si l'on veut, spécifieur. Dans les exemples qui suivent (19) à (23), les verbes lexicaux stand et set dessinent simplement les contours d'une action, alors que UP véhicule l'effet principal. On voit d'abord à quel point la signification de la construction peut varier, et ensuite, comment c'est la signification du morphème UP qui ajuste la pertinence contextuelle de l'expression, tout en demeurant invariante systémiquement, c'est-à-dire sur le plan interlocutif. Dans tous les exemples qui suivent, on retrouve l'expression d'une disruption par rapport à la «norme » à un niveau ou à un autre ${ }^{42}$. Ainsi, en (19), l'action stand perturbe un déroulement normal ou attendu :

19. We were to have dinner together but she stood me up "On devait dîner ensemble mais elle m'a fait faux bond» (le sujet she a agi à l'encontre de ce qui était prévu ; son action, qui a consisté à laisser le locuteur " planté » sur place, a interféré avec ses attentes $\left.{ }^{43}\right)$.

Avec set, UP peut impliquer une activité organisatrice (« organiser, arranger, mettre sur pied ») et une sur-mobilisation typique de $\mathrm{C} 1$ :

20. An anti-terrorist squad was set up « Une équipe antiterroriste a été mise sur pied »

21. She set up her own business " Elle a fondé sa propre entreprise »

22. Let's set up a meeting « Organisons une réunion »

80 Mais la configuration $\mathrm{C} 1$, on s'en souvient, est structurellement différentielle et oppositionnelle. Comme on peut s'y attendre, la mobilisation suffit à déclencher UP, mais dans ce cadre différentiel, la particule peut se teinter volontiers de valeurs négatives dans les rapports interpersonnels (agir à l'opposé des attentes ordinaires de quelqu'un, voire à son encontre) :

23. Somebody set me up/It was a setup "Quelqu'un m'a piégé / C'était un piège »

81 Hors contexte, ces expressions ont une signification pratiquement indécidable. Ainsi, (20) peut aussi bien signifier que l'équipe anti-terroriste a été « constituée, mise sur pied » ou 
bien "prise au piège ", dans une embuscade par exemple. Ce qui compte dans ces emplois de UP, en revanche, c'est toujours la valeur disruptive de C1 que tout anglophone reconnait immédiatement, et qui lui permet d'inférer la bonne interprétation par son insertion contextuelle. Ceci explique qu'on peut trouver des collocations relativement stables ou au contraire plus spontanées. Ainsi, dans l'exemple suivant, tiré du film No Country for Old men, le personnage Llewelyn revient à son motel en taxi et voit une voiture inhabituelle et inquiétante garée sur le parking devant l'alignement des chambres. Il demande alors au chauffeur de ne pas s'arrêter à l'endroit prévu :

25. Don't stop. Just ride me up past those rooms « Ne vous arrêtez pas. Avancez plus loin après les chambres »

Tout d'abord on observe que le verbe lexical ride est, dans le cadre général d'une expression qui décrit le mouvement d'un véhicule, imagé et relativement secondaire (variable sans conséquence majeure). Llewelyn aurait pu employer drive ou take. Quant à UP, sa signification précise est difficile à déterminer, mais on observe que Llewelyn dit au chauffeur de changer la trajectoire initiale du taxi. UP traduit donc une composante essentielle de la signification dans le contexte: le changement de programme dû à la présence de l'autre voiture. Ce sens n'apparaîtrait pas si UP n'était pas employé (Just ride me past those rooms) : en tant que particule, UP apporte la disruption qui le caractérise en propre. L'analyse interlocutive de UP permet de comprendre cette nuance contextuelle très fine qui n'est même pas traduisible en français.

Comme nous l'avons précisé, nous nous centrons sur UP en tant que particule (adverbiale) dans les phrasal verbs mais le sens de contrariété par rapport au cours attendu des choses se retrouve également pour UP utilisé en tant que préfixe comme dans le verbe upend « renverser », au sens propre :

25. I upended my bag to empty it " J'ai retourné mon sac pour le vider » donc upend comme signifiant un mouvement vers le haut. Mais ce serait une erreur, car il faudrait d'abord expliquer pourquoi end impliquerait le bas. En tout état de cause, il y a un ingrédient déterminant qui est l'inversion de la position normale du sac. Dans un sens figuré, on retrouve le même ingrédient :

26. Upend commonly-accepted principles «bousculer les principes communément $\operatorname{admis}^{44}$ »

La valeur de disruption par rapport à la norme permet aussi d'expliquer des cas réputés problématiques, quand coexiste face au verbe avec UP le même verbe sans UP (par exemple end vs end up ou use vs use up). End (" fin ») exprimant déjà, par son sémantisme, l'idée de complétude et de résultat atteint, il est souvent considéré (notamment par les tenants de la thèse aspectuelle du " completive UP ») que la particule est redondante par rapport au sens du verbe et qu'il n'y a donc aucune différence entre les deux. Or, si l'on se base sur des corpus d'emplois authentiques, on constate très vite que ce n'est pas le cas. Les emplois de end up sont réservés au cas où la fin de l'événement est présentée comme une tournure inattendue ou imprévisible. Ce sens d'inattendu (unexpectedness) pour end up est très bien illustré par les exemples que fournit le dictionnaire en ligne Linguee qui pourtant, à l'entrée UP, continue à indiquer comme sens premier « en haut » ou " là-haut ${ }^{45}$ ", alors qu'une prise en compte de ses propres exemples aurait permis de promouvoir davantage le sens disruptif. Il s'agit en effet à chaque fois très clairement d'un résultat qui va à l'encontre du résultat souhaité (nous soulignons dans les énoncés ci-dessous les

Corela, 17-1 | 2019 
éléments lexicaux qui confirment cette interprétation; les traductions sont celles de Linguee) :

27. What you will end up with is generating much more dangerous, much more violent individuals «Au bout du compte, on ne fera que générer des comportements beaucoup plus dangereux et violents »

278. Does he not believe this will instead send the young person to prison, where he will refine his methods and end up doing even more damage? « Ne croit-il pas que cela lui permettra plutôt d'aller en prison, de raffiner ses méthodes et de faire encore plus de dommages?»

29. Under the guise of bolstering democracy, Bill C-251 can end up doing the reverse «Sous le couvert de renforcer la démocratie, le projet de loi C-251 peut comporter des conséquences inverses ${ }^{46}$,

30. Often despite what you try to do, what you start out trying to do once you get through the process you end up doing something different "Souvent, en dépit de ce qu'on essaie de faire, de ce qu'on essaie d'amorcer, une fois qu'on a traversé tout le processus, on se retrouve avec quelque chose de tout à fait différent »

31. (...) concern that by wanting to do too much Unesco may end up doing nothing « (...) la crainte qu'à vouloir trop en faire, l'UNESCO risque, finalement, de ne rien faire "

32. They end up affecting all the other courts and they end up doing us a disservice "Elles finissent par atteindre toutes les autres cours et par nous faire du tort »

33. Birds can become injured or die when they end up in the wings of a wind turbin « Les oiseaux peuvent se blesser ou mourir lorsqu'ils heurtent ${ }^{47}$ les ailes des éoliennes »

Dans tous ces exemples, ce qu'exprime UP, c'est que les choses prennent ou ont pris ou vont sûrement prendre une tournure anormale, non prévue ou non souhaitée. Le résultat à chaque fois est l'inverse du résultat attendu ou visé, ce qui contredit la thèse selon laquelle UP serait marqueur de télicité. Le Cobuild Advanced English Dictionary (dictionnaire en ligne), bien que n'expliquant pas le lien entre cette interprétation de UP dans end up et d'autres interprétations, note à juste titre If you end up doing something or end up in a particular state you do that thing or get into that state even though you did not originally intend to ( «Si vous finissez [end up] par faire quelque chose ou finissez dans un certain état, vous faites cette chose ou vous vous retrouvez dans cet état alors que ce n'est pas ce que vous souhaitiez au départ ») :

34. The result was that the engine ended up at the bottom of the canal « Le résultat est que le moteur a fini au fond du canal »

35. If you don't know what you want, you might end up getting something you don't want «Si tu ne sais pas ce que tu veux, tu risques de te retrouver avec quelque chose dont tu ne veux pas "

36. Every time they went dancing they ended up in a bad mood "Chaque fois qu'ils allaient danser, ça se terminait dans la mauvaise humeur »

Mais, comme nous l'avons déjà souligné, l'interprétation en termes positifs ou négatifs est uniquement affaire d'inférence contextuelle. Ainsi end up en (37) évoque une fin tout-àfait "positive ", UP soulignant seulement l'inattendu de la découverte d'une superbe plage après le désert. UP se contente alors de marquer un contraste :

37. The sand from the desert ends up on a wonderful beach « Le sable du désert cède la place à une superbe plage » (Trip Advisor Reviews Mars 2017).

En (38) ci-dessous, on ne peut pas exactement savoir - à défaut d'indications sur la situation précise et sur les indices prosodiques et kinésiques - en quels termes interpréter les achats de vêtements. On voit cependant que UP met bien en valeur un rapport significatif pour le locuteur entre une situation de départ et une situation d'arrivée. Là encore le contraste est déterminé par la particule : 
38. We visited the wonderful tailors in Khaolak and ended up buying a suit, spare trousers and four shirts " On a rendu visite aux merveilleux tailleurs de Khaolak et on s'est (finalement) retrouvés avec un costume, un pantalon de rechange et quatre chemises » (Trip Advisor Reviews mars 2017) exclu d'y voir un sens perfectif ou télique : ce que marqueraient ces adverbes, c'est, de la même manière que UP, un rapport contrastif entre un état d'avant prévisible et un état d'après inattendu ou spectaculaire. La forme ne donne pas de norme pré-établie (socialement partagée ou présupposée) mais agit comme signal pour la calculer.

Quant aux cas d'emploi de end sans UP - comme le montrent les exemples ci-dessous - il s'agit simplement pour le locuteur d'évoquer factuellement la fin d'un procès ou d'un état. C'est bien sûr toujours le locuteur qui décide de l'angle d'observation du procès :

39. There's no doubt that children from broken homes are twice as likely to have marriages that end in divorce "On sait que les enfants dont les parents ont divorcé sont deux fois plus susceptibles de divorcer eux-mêmes plus tard »

40.In a country where half of all marriages end in divorce, why get married at all ? « Dans un pays où la moitié des mariages finissent par un divorce, pourquoi se marier? "

Le divorce est présenté en (39) et (40) comme un fait statistique, sans commentaire ou jugement de la part du locuteur. La tonalité est factuelle. UP introduirait en plus un jugement moral, en marquant une disruption par rapport à une normalité revendiquée par le locuteur et / ou son public La qualité précise du jugement, positive ou négative, est comme toujours entièrement tributaire du contexte.

L'exemple ci-dessous présente, comme end / end up, un cas d'alternance possible entre le verbe sans particule (use) et avec particule (use up) :

41. On Saturday morning Ruth prepared a proper breakfast for Andy and Nicola. She used up all the eggs that should have lasted through until Thursday - the day that the fresh egg deliveries were made at the local shop- and all the bacon in the house « Le samedi matin, Ruth cuisina un vrai petit-déjeuner pour Andy et Nicola. Elle utilisa (employa à cette fin) toute la provision d'œufs qui devait (pourtant) durer jusqu'au jeudi - le jour où les œufs frais étaient livrés à l'épicerie du coin - et tout le bacon de la maison » (F. Weldon, The Life and Loves of a She-Devil, Pantheon Books, 1983 : 58)

Les défenseurs de la thèse aspectuelle verraient sûrement dans ce UP une parfaite illustration du sens de complétude. Mais use sans UP suffirait à exprimer que, comme cela est explicitement dit lexicalement dans l'énoncé (all the eggs, all the bacon in the house), tous les œufs, jusqu'au dernier, et tout le bacon de la maison ont été utilisés. UP insiste nettement en revanche sur la quantité anormale d'œufs et de bacon utilisés pour un seul petit-déjeuner. Dans l'exemple suivant, de la même manière, le locuteur souligne avec UP qu'il a déjà utilisé tous ses congés alors qu'on est seulement en août :

42. I've used up my holiday entitlement and it's only August «J'ai déjà utilisé tous mes congés alors qu'on est seulement en août "

On trouve aussi invariablement use up dans le titre des livres de recettes pour «accommoder » les restes:

43. How to use up leftovers/How to use up stale bread/chicken «L'art d'accommoder les restes / Que faire du pain rassis / des restes de poulet?»

Il s'agit dans ce cas d'inverser le cours des choses en donnant aux restes une seconde vie. Le côté dynamique et créatif que UP, comme nous l'avons expliqué, permet d'exprimer 
conformément aux caractéristiques de $\mathrm{C} 1$, est également exploité ici (redonner une utilité à des restes autrement condamnés à la poubelle).

Nous pouvons reprendre le cas de bind up et break up, cités par Denison comme une illustration des emplois contradictoires de UP (cf. supra, partie 1). Bind signifie « unir, associer, (re)lier ». UP soulignera en contexte un changement fort par rapport à un état d'équilibre. On aura par exemple to be bound up in some research et pas seulement bound pour souligner l'engagement spécial ou exclusif du sujet et sa mobilisation. Bind up peut aussi s'appliquer au pansement d'une blessure (bind up a wound) qui remédie à un problème. Break up est particulièrement intéressant : break signifiant une fracture, ou une pause, etc, UP ajoute une dimension contrastive par rapport à un état présumé équilibré ou normatif. C'est ainsi qu'un couple ou une famille peut connaître un état momentané de séparation (break) mais cette séparation peut aussi se transformer en rupture, nouvel état global disjoint opposé à l'état «normal» (break up $\left.{ }^{48}\right)$. C'est la raison pour laquelle la récréation des enfants à l'école est simplement désignée par break, alors qu'on trouve break up pour les vacances, par opposition au statut «normal » des écoliers (exemples de Reverso, en ligne, entrée break) :

44. The school breaks up next week/The children have already broken up «Les cours finissent la semaine prochaine / Les enfants sont déjà en vacances » (déjà soulignant la rupture de situation)

On voit que UP, par la disruption contrastive, globalise des changements d'états ou de situations. La raison en est que la particule installe les procès au niveau des normes individuelles ou sociétales. Ce faisant, la particule provoque une totalisation, en apportant au procès une dimension globale et définitive. C'est de là que viennent des effets habituellement décrits comme aspectuels. Ces nuances sont souvent extrêmement fines alors que, sur le plan communicationnel, elles sont d'une très grande portée. Seul un modèle interlocutif est à même de les mettre en évidence et de les valoriser.

Le verbe put up offre un éventail intéressant de la déclinaison d'effets de sens variés qui découlent tous de la valeur C1 de UP. En (45) ci-dessous, il s'agit de se mobiliser, prendre les choses en main, pour héberger quelqu'un à titre exceptionnel :

45. Fortunately they put me up for the night « Heureusement, ils m'ont hébergé pour la nuit »

Selon les contextes appropriés, on pourra trouver la valeur différentielle ou discordancielle de C1, qui sera plus ou moins mise en avant. Ainsi, (46) et (47) peuvent singulièrement s'appliquer à des cas où il sera question d'une association problématique :

46. Put up with someone "supporter quelqu'un, arriver à s'entendre avec cette personne "

47. He has a lot to put up with «Il doit faire face à beaucoup de difficultés »

La sur-mobilisation caractéristique de C1 (prise en main) pourra dans certains contextes se prêter à l'expression d'une sur-mobilisation du sujet pouvant être interprétée dans le sens d'un engagement très fort, d'une décision prise ${ }^{49}$ :

48. He put up for pre-election «Il s'est porté candidat pour un nouveau mandat »

49. Put up money for a project "mobiliser, lever des fonds" (on appréciera la métaphore « lever » en français)

101 UP marque de façon générale la "prise en main ». D’autres exemples sont remarquables de ce point de vue comme take up (" entreprendre»), start up (" démarrer, organiser, construire »), meet up (« rencontre qu'on organise expressément »), pull one's socks up (« se 
remuer ", "se retrousser les manches ${ }^{50}$ ). Think up s'applique à une entreprise globale de réflexion et d'innovation, et non simplement à une pensée.

L'identité et la conformité absolue et nécessaire prévalant au contraire en C2, les formes relevant de cette configuration sont par contraste en affinité avec tout ce qui s'apparente à une notion de passivité et d'extinction (put down). Sans creuser davantage cette opposition, on peut simplement rappeler que les formes C2 s'appliquent au sens du courant (downstream) alors que les formes $\mathrm{C} 1$ spécifient le contre-courant :

50. Swim, row upstream " nager, ramer à contre-courant, remonter ${ }^{51}$ le courant "

Back up est très proche, avec le sens « faire marche arrière ». Mais il peut aussi signifier « soutenir (un candidat)", «s'engager (pour lui)» ou encore « étayer (un bâtiment, un argument)».

La collocation make up offre comme put up une variété d'interprétations qui peut surprendre mais qui découlent toutes des caractéristiques de $\mathrm{C} 1$ dont relève UP. L'inventivité est mise en avant dans make up au sens d'«inventer (une histoire, des excuses) », voire " préparer (un lit, un paquet) », ou " rédiger (une ordonnance) ». Make up peut aussi renvoyer à la fragmentation intérieure d'un ensemble ( = constitute) comme dans :

51. Right-wing MPs make up two-thirds of the committee «Les deux tiers de la commission sont composés de députés de droite "

Cette fragmentation du grand ensemble (par exemple ici le comité) est corrélative de la promotion globalisante de la partie elle-même (les députés de droite) en (sous-)ensemble constituant. Dans le sens de "se maquiller » pour make up, prédomine le changement par rapport à l'apparence normale (voir dress up) mais aussi le sens de « refaire un visage, une apparence ». Dans make up with someone "se rabibocher / se réconcilier avec quelqu'un ", le sujet contrarie une situation estimée négative. Cet aspect réactif s'observe aussi dans les cas de correction ou de remédiation comme make up (for) lost time/lost ground (rattraper le temps perdu, rattraper son retard). L'exemple ci-dessous montre une variation avec play:

52. [Sylvie Palot, fille de protestants, est attirée par un jeune homme qu'elle vient de rencontrer au marché, et elle réfléchit à cette rencontre]

She already had one admirer. Among her parents' acquaintances were the Mauriac family. Father and son were both short and played up to it by being cheery wisecracking chaps: the father, Luc, was a charmer, and everyone liked him [...] but the son, Georges, who was Sylvie's admirer, was a pale imitation, all poor jokes and clumsy sallies « Elle avait déjà un soupirant. Parmi les connaissances de ses parents figurait la famille Mauriac. Le père et le fils étaient petits tous deux, mais ils compensaient ce défaut par leur jovialité et leur caractère blagueur: le père, Luc, était un charmeur, et tout le monde l'appréciait [...] mais le fils, Georges, le soupirant de Sylvie, en était une pâle imitation, qui n'était que plaisanteries médiocres et saillies maladroites ${ }^{52}$ " (Ken Follett, A Column of Fire, Pan Books, 2017 : 57-58, c'est nous qui soulignons)

Pour close, il est généralement relevé que close down s'applique à une fermeture définitive. La configuration C2 de DOWN en effet impose un cours inéluctable des choses, et close down ne laisse pas envisager la possibilité d'une option alternative. Close up s'appliquerait à l'inverse à un acte de fermeture non définitif :

53. I thought it best to close up and secure the inventory «Je pensais qu'il serait mieux de fermer pour sécuriser le stock » (Reverso) notion de disruption par rapport à une situation qui a normalement cours. En (53), close 
up peut s'appliquer à une fermeture momentanée ou occasionnelle, mais on voit que cette clôture est en fait centrée sur le besoin de sécurité, et, à la limite, close up ici pourrait très bien signifier la même clôture définitive que close down: par exemple, si une inondation condamne une boutique à fermer, close up pourra parfaitement être employé, pour souligner la disruption, alors que close down s'appliquera à une fermeture simplement donnée comme inéluctable, ou naturelle, ou normale.

On peut faire les mêmes observations pour slow up et slow down (exemples (54) et (55) donnés par un blog internet) :

54. If I run with Christina, she tends to slow me down « Quand je cours avec Christina, elle a tendance à me ralentir ».

109 Le ralentissement est une conséquence naturelle de la lenteur de Christina: c'est involontairement et sans même s'en rendre compte qu'elle ralentit sa partenaire. En revanche, slow up s'appliquera à une décision locale et réactive :

55. We slowed up when we saw the police "Nous avons ralenti en voyant la police"

110 On a le même phénomène avec rest dans le cas où l'interlocuteur n'a pas envie de se reposer, ou bien quand on conseille à quelqu'un de se réserver une période de repos, soit localement en vue d'une préparation intensive, soit quand cette personne n'a pas forcément prévu de le faire :

56. You should rest up «Tu devrais prendre un peu de repos » (noter l'expression française avec « prendre »)

111 Les effets de sur-mobilisation pour contrarier, inverser et contrecarrer le cours des choses expliquent la fréquence de UP à l'impératif dans les cas où il s'agit de pousser l'interlocuteur à faire quelque chose que, naturellement, il ne ferait pas. On peut ranger dans cette catégorie les cas de Eat up! ou Drink up !, Listen up !, Wait up !, Hurry up !, etc. Ce n'est pas la complétude qui est exprimée ici par UP. Eat up! ne signifie pas «Finis ton assiette ! », bien que ce soit la traduction la plus commode et la plus répandue (voir supra à propos de Denison 1985). Il s'agit d'une demande d'activation, de mise en route d'un processus qui inverse un cours des choses qu'on ne souhaite pas. C'est la raison pour laquelle on dit généralement Hurry up! plutôt que Hurry! seul (tout-à-fait possible par ailleurs).

112 La même observation peut être faite à propos de Time is up! « c'est l'heure, c'est fini » qui avertit que l'heure de fin d'une épreuve est arrivée. Que l'épreuve prenne fin à ce moment là est une inférence contextuelle. Ce qui compte, c'est la rupture du cours des choses. Le nom du mouvement social Time's up, lancé en 2018 à Hollywood suite à l'affaire Weinstein, est une très belle illustration de la disruption typique de C1: Time's up « C'est l'heure » signifie la fin du harcèlement sexuel en annonçant des temps nouveaux où ces agissements ne seront plus tolérés. Le sens est bien «L'heure nouvelle (différente) est arrivée », et non pas «l'heure ancienne (conservatrice) est finie ».

113 Dans l'exemple ci-dessous, le personnage Jared veut encourager son interlocuteur ivre à boire le café qu'il lui a commandé pour le dessoûler. En aucun cas il ne lui demande de le « finir ». Il le pousse à agir, à combattre son ivresse et sa torpeur :

57. 'I'm Sam.' His inebriated tongue slurred the 's', and his handshake had a wave to it, but the sentiment was there. Jared slid the coffee in front of Sam and encouraged him to drink up " 'Moi, c'est Sam'. Le 's' chuintait sous l'effet de l'alcool, et la poignée de main tremblait un peu, mais le sentiment y était. Jared glissa le café devant Sam et l'encouragea à le boire » (ce serait un contresens de traduire par 
«l'encouragea à finir» le café) (Shirley Jump, Sweetheart Lost and Found,

Harlequin, 2008, p. 13).

114 Shut up ! est l'expression d'un antagonisme car il s'agit de contraindre l'interlocuteur à changer son comportement et se taire. C'est un cas d'adversité maximale explicite, et un vecteur polémique. De même, look up implique qu'on doit réorienter le regard dans un sens ciblé, autre que le mouvement naturel. De plus, look up ne signifie pas simplement « regarder» (look) mais évoque tout un processus global et un travail.

Reprenons pour terminer ce tour d'horizon de UP, le cas de abandon/give up : le lecteur aura compris que les deux verbes signifient la même chose à une nuance importante près, qui est que UP apporte un élément contextuel de disruption. Give up implique donc un renoncement de manière explicite, ce que ne fait pas directement abandon. On pourra comparer l'expression Abandon ship! «Quittez le navire!», qui est la formule officielle et codifiée dans le cas d'un naufrage, avec le dernier ordre de James Lawrence, capitaine de la frégate américaine Chesapeake en 1813. Lawrence ordonne de ne pas quitter le navire afin de ne pas le livrer aux Anglais et dit : Don't give up the ship !

\section{Conclusion}

Nous espérons avoir montré dans cet article qu'il était possible de traiter de manière unifiée l'ensemble des emplois de UP, en évitant la rubrique "autres emplois inclassables ». La condition pour ce faire est de traiter du langage comme d'un système à part entière, auto-organisé et auto-référent. Cette auto-référence invite à mettre en débat certaines relations à l'environnement, trop souvent et trop facilement réputées déterminantes dans la structuration du système. Parmi ces relations, la place prioritaire accordée aux relations spatiales, en raison de leur simplicité et de leur commodité, en raison surtout de leur entrelacement constant avec les relations non spatiales, doit être réévaluée. L'espace, comme le temps, sont des éléments du contexte et de l'environnement. Or, par définition, le système ne peut pas connaître d'emblée cet environnement, et c'est le système qui tisse les liens avec l'environnement, pas l'inverse. Les théories localistes renversent tout le processus constructif du système en le rendant sinon dépendant, du moins directement sensible aux déterminations extérieures ${ }^{53}$. Il est probable que notre comportement organique et psychologique est assez lisiblement lié aux stimuli environnementaux. En revanche, pour le langage, qui manifeste une structuration complexe et très variable, quels que soient ces stimuli, il est plus prudent d'adopter une position systémiste et accorder la priorité à sa structuration propre. La Théorie de la Relation Interlocutive (Douay \& Roulland 2014) est un modèle qui revendique cette auto-référence et essaie d'en tirer toutes les conséquences. En l'occurrence, pour les morphèmes comme UP, il considère par définition qu'il s'agit de valeurs interlocutives, définissant des «cadres réceptifs » ou "configurations » qui orientent et modulent l'interprétation contextuelle.

Il conviendrait de montrer que toutes les particules / prépositions peuvent s'expliquer dans les mêmes termes que UP et s'articulent en sous-systèmes. Opposé à DOWN comme IN s'oppose à OUT, ou ON à OFF, UP est une marque configurationnelle de type $\mathrm{C} 1$, c'est-àdire porteuse de disruption par rapport à une normalité conventionnelle. Au terme de cette démonstration, il apparaîtrait que ces couples morphémiques catégorisent des aspects de l'opération de réplication linguistique (comme IN vs OUT, qui s'applique à une analyse réflexive qui peut se métaphoriser en intériorité). Pour le modèle de la TRI les 
articulations systémiques ne proviennent ni directement du monde, ni des (inter)actions du corps avec / dans le monde. Elles proviennent d'abord des opérations du système luimême sur lui-même. Les distinctions établies par les théories spatialistes sont des interprétations contextuelles et inférentielles de ces opérations systémiques. Autrement dit ce sont les «actions » du système qui modélisent les opérations de localisation. Pour cette raison, c'est toujours la réplication et l'interlocutivité qui sont prioritairement en cause.

\section{BIBLIOGRAPHIE}

Adamczewski, H. (2003) Du nouveau sur les phrasal verbs : UP revisited, Précy-sur-Oise, EMA.

Adamczewski, H. \& J.P. Gabilan (1992) Les clés de la grammaire anglaise, Paris, Armand Colin.

Bolinger, D. (1971) The Phrasal Verb in English, Cambridge, Harvard University Press.

Brinton, L. J. (1988) The Development of English Aspectual Systems, Cambridge, Cambridge University Press.

Busuttil, P. (1995) "Phrasal verbs : A contribution towards a more accurate definition", Asp [online], 7-10 1995, online since 30 July 2013.

Cadiot, P. (2002) «Éléments d'une critique de la notion de préposition spatiale », Syntaxe et sémantique, 1/3, 117-129.

Cadiot, P. \& Lebas, F. (2003) « Monter et la constitution extrinsèque du référent », Langages 150, 9-30.

Constantin de Chanay, H. \& Rémi-Giraud, S. (2002) « Espèces d'espaces » : approche linguistique et sémiotique de la métaphore ", Mots. Les langages du politique [En ligne] 68, consulté le 28 février 2019, URL : http://journals.openedition.org/mots/7013.

Culioli, A. (1976) « Transcription par les étudiants du séminaire de D.E.A. », Recherches en linguistique, Théorie des opérations énonciatives, Département de Recherches Linguistiques, Université Paris 7.

Culioli, A. (1985) Notes du séminaire de D.E.A. 1983-1984, Poitiers.

Culioli, A. (1997) « A propos de la notion », in Rivière, C. \& Groussier, M.-L. (dir) (1997) La notion. Actes du colloque tenu à l'U.F.R. d'Études Anglophones, Université Paris 7 , Paris, Ophrys, 9-24.

Denison, D. (1985) “The origins of completive up in English”, Neuphilologische Mitteilungen 86, $37-61$.

Desclés, J-P. (2000) « Imparfait narratif et imparfait de nouvel état en français », colloque de Cracovie, [En ligne] consulté en mars 2019, URL : http://lalic.paris-sorbonne.fr/PUBLICATIONS/ publications.php?annee $=2000$.

Douay, C. (2000) Eléments pour une théorie de l'interlocution, Rennes, Presses Universitaires de Rennes. 
Douay, C. \& Roulland, D. (2014) Théorie de la relation interlocutive. Sens, signe, réplication, Limoges, Editions Lambert-Lucas (réédition en 2018).

Douay, C. \& Roulland, D. (2016) « L'auxiliaire DO dans les affirmations non-polémiques en anglais », Testi e Lingaggi 10, 56-67.

Dru, J-M. (1996) New : 15 approches disruptives de l'innovation, Montreuil, Ed. Pearson.

Fagard, B. \& Stocik, D. (2012) «Introduction générale : langue, espace, cognition », Corela [En ligne] HS-12, consulté le 05 mars 2019, URL : http://journals.openedition.org/corela/2720.

Fortis, J-M. (2010) « De la grammaire générative à la grammaire cognitive : origine et formation de la théorie de Ronald Langacker », Histoire Épistémologie Langage 32/II, 109-149.

Franckel, J-J. \& Paillard, D. (1998) « Aspects de la théorie d'Antoine Culioli », Langages 129, 52-63.

Gardiner, A. H. (1932) The Theory of Speech and Language, Oxford, The Clarendon Press, $2^{\mathrm{e}}$ éd. 1951, traduction française par Douay, C. Langage et acte de langage. Aux sources de la pragmatique, Villeneuve d'Ascq, Presses Universitaires de Lille, 1989.

Getliffe, P. (1999) Verbes prépositionnels et verbes à particule, Paris, Ellipses.

Grossmann, F. (2017) «La notion d'évidence et son expression linguistique dans la rhétorique scientifique », Travaux Neuchâtelois de linguistique 65, 37-52.

Jacques, F. (1979) Dialogiques : Recherches logiques sur le dialogue, Paris, FeniXX.

Jalenques, P. (2002) «Étude sémantique du préfixe RE en français contemporain : à propos de plusieurs débats actuels en morphologie dérivationnelle », Langue française 133, 74-90.

Kleiber, G. (2001) « Sur le sens du sens : objectivisme et constructivisme », in Keller, D., Durafour, J.-P., Bonnot, J.-F. \& Sock, R. (éds.) Percevoir : monde et langage, Bruxelles, Mardaga, 335-370.

Lakoff, G. (1987) Women, Fire and Dangerous Things : What Categories Reveal about the Mind, Chicago, University of Chicago Press.

Lakoff, G. \& Johnson, M. (2003) Les Métaphores dans la vie quotidienne, Paris, Minuit, traduction française de Metaphors We Live By, Chicago, Chicago University Press, -1980.

Langacker R. (1982). “Space Grammar, Analysability, and the English Passive”, Language 58/1 (March), 22-80.

Langacker, R. (2008) Cognitive Grammar. A Basic Introduction, Oxford, Oxford University Press.

Lapaire, J-R. (2017) « Grammaire cognitive des prépositions : épistémologie et applications », Corela [En ligne] HS-22, consulté le 05 mars 2019, URL : http://journals.openedition.org/ corela/5003.

Lapaire, J-R. \& Rotgé, W. (1993) Séminaire pratique de linguistique anglaise, Toulouse, Presses Universitaires du Mirail.

Lapaire, J-R. \& Rotgé, W. (2004) Réussir le commentaire grammatical de textes, Paris, Ellipses.

Larsson, B. (1997) Le Bon sens commun : Remarques sur le rôle de la (re)cognition intersubjective dans l'épistémologie et l'ontologie du sens, Études Romanes de Lund, Vol 57, Lund University Press.

Larsson, B. (2008) « Le sens commun ou la sémantique comme science de l'intersubjectivité humaine ", Langages 170, 28-40.

Laurendeau, P. (1998) «Théorie des opérations énonciatives et représentations : la référenciation ", Cahiers de praxématique 31, 91-114. 
McIntyre, A. (2002) “Idiosyncrasy in particle verbs”, in Dehé \& al. (eds.) Verb-particle explorations, Berlin, Mouton de Gruyter, 97-118.

McIntyre, A. (2003) "Preverbs, argument linking and verb semantics. Germanic Prefixes and Particles”, Yearbook of Morphology 2003, 119-144.

Nemo, F. (2014) «Interprétabilité ou grammaticalité ? Les listèmes comme interface entre sémantique et morphologie ", Revue de Sémantique et Pragmatique 35-36, 105-144.

Quayle, N. (1994) UP et le verbe à particule en anglais contemporain, Lille, Presses Universitaires de Lille.

Riguel, E. (2015) « Les phrasal verbs : usage et acquisition », Textes et Contextes, Université de Bourgogne, Centre Interlangues TIL, <halshs-01246384>

Roulland, D. (2017) « Langage et réplication », Intellectica 68, 69-97.

Rousseau A. (1993) « Espace, référence, représentation. Réflexions sur quelques conceptualisations de l'espace », Faits de langues n 1, Motivation et iconicité, 151-162.

Saussure, F. de (1973) Cours de Linguistique Générale, publié par Charles Bally et Albert Sechehaye, avec la collaboration d'Albert Riedlinger ; édition critique préparée par Tullio de Mauro, Paris, Payot, -1916.

Schmoll, P. (1996) « Production et interprétation du sens : la notion de contexte est-elle opératoire? ", Scolia 6, 235-255.

Toupin, F. (2006) « Les sens procéduraux intersubjectifs de la particule UP », Corela [En ligne] 4-2, consulté le 13 octobre 2016, URL : http://corela.revues.org/469

Trebits, A. (2009) « The most frequent phrasal verbs in English language EU documents - A corpus-based analysis and its implications", System 37, 470-481, [En ligne], consulté en mars 2019, URL : www.sciencedirect.com. (Budapest, Eötvös Loránd University (ELTE), Department of English Applied Linguistics, Language Pedagogy PhD Program, pdf).

Vandeloise, C. (1987) «La préposition à et le principe d'anticipation », Langue française 76, 77-111.

Victorri B. (1999) « Le sens grammatical », Langages 136, 85-105.

Victorri, B. (2010) « Le localisme à l'épreuve du verbe aller », Corela [En ligne], HS-7, consulté le 06 mars 2019, URL : http://journals.openedition.org/corela/1009.

Weill, I. (2009) « Re- dans tous ses états, un « préfixe » marquant l'aspect implicatif », Linx 60, 119-140, [En ligne] 60, consulté le 23 février 2019, URL : http://linx.revues.org/705.

\section{NOTES}

1. Nous remercions nos relecteurs de CORELA, dont les observations constructives nous ont permis de préciser les points de vue développés ici.

2. Nous centrerons notre étude sur les cas où l'interprétation de UP est généralement considérée la plus problématique, à savoir son emploi comme particule verbale dans les verbes dits composés ou à particule (phrasal verbs). En ce qui concerne la catégorisation des particules verbales comme "adverbes» ou "prépositions» ou encore "prépositions adverbiales» ou " adverbes prépositionnels ", nous renvoyons le lecteur à l'analyse très éclairante de P. Busuttil (1995), qui suggère pour les phrasal verbs l'appellation adverbed verbs ("verbes adverbiés »). Défendant le principe d'un invariant des formes grammaticales, quel(s) que soi(en)t le(s) statut(s) syntaxique(s) qu'elles peuvent acquérir en discours, la valeur systémique que nous définirons 
pour UP vaut pour tous ses emplois, compte tenu, bien entendu, des contraintes et latitudes liées à chacune de ces catégories.

3. Cf. Denison (1985: 2) : “Up presents a complex history, possibly including significant foreign influence, and it is the Aktionsart particle par excellence-in fact it is the particle occurring in the largest number of phrasal verbs". Voir aussi Trebits (2009).

4. L'expression, à l'origine, était «tomber sous les sens » et s'employait pour signifier « qui n'a pas besoin d'être compris, qui est directement observable ». Voir à ce sujet le très intéressant article de F. Grossmann (2017).

5. Nous reviendrons sur les exemples que nous donnons dans ce premier paragraphe dans notre analyse discursive . Tous nos exemples sont authentiques (source indiquée) même si dans des cas d'emploi très courants comme, par exemple What's up ?, nous avons estimé que la mention d'une source spécifique n'était pas nécessaire.

6. L'un de nos aimables relecteurs déplore le fait que nous ne tenions aucun compte de «la primarité chronologique (historique) du sens spatial». Nous devrions selon lui "absolument distinguer cette forme de primarité, qui est chronologique, factuelle (prouvée par les textes) de la primarité au sens d'une prépondérance accordée au spatial, dans une théorie donnée, par rapport aux autres sens attestés d'un marqueur (qui, elle peut être contestée) ». L. Brinton (1988 : 197), à l'ouvrage de laquelle nous renvoie notre relecteur, affirme certes que «the prefixes and particles occur first in contexts in which spatial meanings are primary, with verbs of motion or of physical action». Nous ne contestons évidemment pas que UP ait des valeurs spatiales ni même que ce soient ces valeurs qui aient été au départ les plus visibles. Mais si on se réfère à $P$. Gettliffe (1999 : 72) auquel nous renvoie également le relecteur, en vieil-anglais, même s'ils sont plus rares, des emplois classés par l'auteur dans la catégorie «emplois figurés » ou «idiomatiques » coexistent avec le sens considéré comme "littéral, c'est-à-dire spatial». N. Quayle (1994 : 127) rappelle que Curme (1914 : 328) mentionne en vieil-anglais des sens « figurés » à côté des sens " concrets » et "spatiaux ». Nous considérons que ces sens tenus pour figurés et, pour certains, chronologiquement seconds, faisaient déjà partie de la panoplie des sens de «surface » de UP et se sont trouvés simplement moins activés ou exploités, du moins dans les traces écrites dont nous disposons du vieil-anglais. La tendance très courante à faire tout commencer par le concret, et à rendre le relationnel et l'abstrait supérieurs et tardifs, s'apparente à un primitivisme qui nous semble très suspect. Pour notre part, nous croyons (car ce ne peut être prouvé ni dans un sens ni dans un autre) que c'est le langage qui a permis ou au minimum accompagné l'abstraction, et ce dès ses premiers rudiments.

7. Voir aussi Cadiot \& Lebas (2003).

8. La traduction dans une autre langue peut dans certains cas corroborer une interprétation, et nous en verrons des exemples pour UP entre l'anglais et le français, mais il convient de se méfier grandement de l'évidence, aussi, en cette matière.

9. Voir infra pour d'autres interprétations possibles de back up. ("soutenir(un candidat)», « s'engager (pour lui) » ou encore « étayer »(un bâtiment, un argument)).

10. Put somebody up signifie ici « héberger provisoirement quelqu'un ». Il ne s'agit là que d'une interprétation possible de put up. On notera que la logique spatiale ferait plutôt attendre DOWN vu qu'il s'agit d'offrir à quelqu'un la possibilité de poser ses affaires et de se coucher. Nous revenons sur ce verbe dans notre article, et aussi sur rest up qui signifie « se reposer ».

11. On retrouve la notion de perfectivité, associée à celle de " résultativité », dans l'analyse de UP développée par N. Quayle (1994) dans le cadre de la psychomécanique du langage : la «visée perfective" signifiée selon l'auteur par UP permet de "rendre compte des notions de complétude ou de résultativité qui caractérisent l'emploi de UP dans de nombreux verbes à particule ». Cette « visée perfective » dériverait de ce que N. Quayle considère comme le premier sens «historique» de UP (mouvement vers le haut), sens qui aurait ensuite subi une « dématérialisation qui lui permet de représenter des mouvements latéraux ou d'approche d'une 
limite.»: «En fait, quelle que soit la signification de la particule par rapport au verbe (mouvement vers le haut, approche, résultativité...), il existe, comme apport constant, une notion de perfectivité. Up fonctionne, en quelque sorte, comme un marqueur de perfectivité lexicale aussi bien dans les sens les plus concrets qu'abstraits. Ainsi la particule discute de la limite qui sépare l'opérativité et la résultativité verbales ». (1994: 224).

12. Cette équation entre perfectivité et télicité se rencontre chez de nombreux auteurs. L'un de nos relecteurs nous renvoie à ce propos à l'étude de L. Brinton (1988) qui s'attache à bien distinguer les deux notions. Chez Brinton, résume notre relecteur, "la télicité relève de l' aktionsart, ce qui n'est pas le cas de la perfectivité. La télicité est le fait pour une activité (eat) de recevoir un point final, une borne de droite (eat something up) ; elle n'indique rien de l'atteinte (ou non) de ce point final (endpoint). La perfectivité ou résultativité est le produit d'une combinaison textuelle entre télicité et perfectivité ; c'est par exemple le cas où un procès télique est exprimé dans un énoncé au prétérit ou à l'aspect parfait, ce qui renvoie à l'atteinte effective du point final ». Si cette distinction peut s'avérer utile, par exemple pour les approches s'intéressant à la catégorisation des verbes selon qu'ils sont téliques ou pas, elle s'inscrit dans une problématique référentialiste - problématique non pertinente dans l'approche interlocutive qui est la nôtre. En outre, comme nous le verrons plus loin, elle ne permet pas d'expliquer le sens de UP dans, par exemple, eat up. Il faut reconnaître cependant que, sur le plan théorique, elle présente l'avantage d'éviter les écueils référentialistes avérés de la résultativité au profit d'une opérativité plus cognitive.

13. Voir D. Bolinger (1971: 96 -97). Pour Bolinger, d'une part, un phrasal verb est une unité lexicale qui n'est pas la somme de ses parties (non additive compound or derivative, 1971 : xii), et d'autre part les extensions analogiques sont telles, dans toutes les directions, qu'il est impossible de lui donner une interprétation fixe (1971:6). Il ajoute que «les positions variables de la particule et d'autres facteurs » confèrent au phrasal verb un statut hybride, «à la frontière entre morphologie et syntaxe » $(1971: 111)$.

14. En anglais, le phénomène est tellement extensif qu'on ne peut pas y voir des cas accidentels de synonymie, ou l'effet d'une mode, même si des distinctions de registre existent. Notons en outre, bien que nous n'abordions pas dans la présente étude cette question, que le verbe composé permet également par rapport au verbe simple d'introduire des nuances sémantiques en jouant sur la place syntaxique de la particule. Preuve s'il en fallait que la particule a bien un sens relativement indépendant du verbe.

15. Pour A. McIntyre (2002), les linguistes supposent le plus souvent que les constructions verbeparticule tendent à être non-compositionnelles. Il parle d'un « mythe » selon lequel une particule qui n'a pas de sens spatial ne peut pas se combiner compositionnellement avec un verbe et s'interprète tout au plus dans un sens télique/ perfectif. De nombreuses combinaisons « idiosyncratiques » en apparence, s'avèrent en réalité dérivables du sens de leurs éléments, une thèse qu'il est possible et souhaitable de généraliser. Voir aussi McIntyre (2003: 7) : "The idea that preverbs primarily contribute telicity is probably connected to the idea that Germanic prefixes and particles always yield telic VP's. (...) This myth is refuted by examples like the following, where the in-time test is passed without any event iteration effects : Grandma held down the wrestler for an hour; They kept out the enemy for a day; She slagged off her husband for 10 minutes; I scrubbed down the table for 10 minutes; They beat him up for five minutes; He stared down his opponent for a minute; Fran chatted up Stan for ten minutes ».

16. Notons que «infantiliser» signifiant un abaissement, une diminution de statut, les définitions spatiales feraient plutôt attendre DOWN.

17. C'est quelque peu hâtivement, de notre point de vue, que Laurendeau (1998: §25) déclare par exemple : «la problématique de l'activité de langage est inséparable d'une problématique de la représentation du monde. Comme cette affirmation n'est pas réversible, il y a lieu d'en tirer les conclusions nécessaires sur la non-autonomie de la linguistique comme discipline cognitive ». Il 
n'a jusqu'ici été fourni aucune preuve qu'une quelconque représentation du monde était possible «sans langage» et l'idée ne peut être envisagée qu'au prix d'une définition de l'activité linguistique extrêmement restrictive.

18. Voir à ce sujet A. Rousseau (1993 : 153).

19. Gustave Guillaume considérait que le temps, n'étant pas représentable à partir de lui-même, devait être mentalement "spatialisé ", et prévoyait que la production du discours impliquait un «parcours» du temps opératif des systèmes de langue. Par ailleurs, de façon générale, il tenait l'espace comme une base de représentation plus simple qu'aucune autre, et plus fondamentale, puisque l'espace impliquait selon lui, immédiatement et commodément, une discrétisation primaire que pouvait utiliser la pensée.

20. Un de nos relecteurs rappelle qu'il s'agit en réalité de métonymisation et non de métaphorisation. Cette distinction très utile est pertinemment rappelée. Elle n'a cependant pas d'incidence directe sur notre présent propos, puisque nous entendons seulement souligner qu'il n'y a pas de différence de nature ni de hiérarchie entre les sens spatiaux et les sens figurés. Nous considérons qu'il n'y a pas non plus de primarité diachronique de l'espace.

21. Que le langage soit auto-référentiel n'implique absolument pas qu'il soit indépendant du monde et de la cognition. C'est même l'inverse qui se produit en toute nécessité. L'organisme humain prend conscience du monde et le « comprend» en lui appliquant la logique structurelle du système sémiotique. Si cette logique systémique n'était pas autonome, l'emprise sur le monde serait nulle, contrairement à ce que démontrent quotidiennement les progrès de la cognition. Le langage n'est pas indépendant de notre relation cognitive au mondeprécisément parce qu'il est en lui-même le matériau et la forme de cette relation.

22. Par ailleurs, les grammaires cognitives ne font pas de distinction entre signification et traitement cognitif. Il s'ensuit que la question de la contextualité, et surtout de l'indispensable abstraction des formes par rapport aux divers contextes, devient aiguë au point qu'il est nécessaire de contraindre les opérations par un "maximum de structure » (Fortis 1996). Cette structure doit non seulement être stable et universelle, mais elle doit aussi être externe et préexistante afin de laisser la voie libre aux opérations d'appropriation et de contextualisation, dont on comprendra qu'elles peuvent quasiment toutes se ramener à la métaphorisation. La simplicité et l'économie sont également indispensables car chaque production linguistique implique la réitération des opérations mentales en cause.

23. Un des problèmes qui se pose pour cette approche est la profondeur historique de l'évolution dont il est question. Si on acceptait l'idée que les relations spatiales sont primaires, et l' intersubjectification l'étape ultime dans l'édification du langage humain, les deux plans seraient pratiquement confondus de toute façon dans un passé (pré-)paléolithique inconnaissable. On ne peut absolument pas les dissocier, par exemple comme des sens "propres » et «figurés», $a$ fortiori dans des textes écrits tous très récents au regard de cette grande histoire.

24. "From this follows the important consequence that a physical substitute has to be found whenever anything intellectual or emotional is to be imparted. Such physical substitutes are called signs, are subject to the conditions (1) that they should have a pre-arranged 'meaning', or associated mental equivalence, and (2) that they should be handy objects of sense transferable at will. Any material thing which conforms to these two conditions will serve as a 'sign', and any system of signs is a kind of language" (1932: 67).[De là découle la conséquence importante qu'un substitut physique doit être trouvé chaque fois qu'il s'agit de communiquer quoi que ce soit d'intellelectuel ou d'émotionnel. Ces substituts physiques sont appelés signes et ils sont soumis aux conditions suivantes : (1) ils doivent avoir un sens pré-établi, c'est-à-dire être associés à un équivalent psychique et (2) ils doivent être des objets perceptibles, faciles à utiliser et transférables à volonté. Toute chose matérielle conforme à ces deux conditions peut servir de « signe » et tout système de signes est un type de langage]. 
25. Nous maintiendrons « l'un l'autre » qui, bien que pouvant être jugé redondant et maladroit, insiste sur la mutualité de l'échange et sur le rôle capital de l'interlocuteur.

26. F. Jacques ajoute: "Non qu'il [le langage] nous fasse entrer en nous-même et retrouver quelque esprit unique et commun auquel nous participerions, mais parce qu'il nous concerne, nous transforme en l'autre et lui en nous. Comment aussi il introduit, par des altérations subtiles de son sémantisme, le 'deux en un' et le 'un en deux'. Le dialogue est coextensif aux structures profondes de la parole. » (1979: 48).

27. Au niveau systémique, en raison de la triangulation et de l'objectivation, ces polarités ne sont pas « incarnées » pourrait-on dire. Elles ne désignent en aucune façon des individus empiriques. On pourrait les qualifier de « rôles abstraits ", purement relationnels, d'où ces appellations $\alpha$ et $\beta$ (voir Douay \& Roulland $2014: 138$ ).

28. Cette objectivité repose sur un «pacte " ou " accord» interlocutif qui vise à contrôler au maximum la subjectivité des organismes en communication. Pour B. Walliser (1977), un modèle peut être tenu comme « objectif » s'il est jugé valable par tous les observateurs, c'est-à-dire si son « champ de validité » est le plus étendu possible. Dans le cadre de la TRI, le champ de validité du signe est la relation interlocutive entre les deux observateurs partenaires sous le contrôle commun du signe, si bien que l'objectivité linguistique est toujours un rapport entre l'objectivité (pratique) relative au niveau des interlocuteurs et l'objectivité (théorique) absolue du signe.

29. Larsson $(1997,2008)$ écrit dans son résumé introductif de (1997) : «Le sens, pour être, doit contenir une cognition ou une conceptualisation intersubjectivement reconnue, codifiée et mémorisée par au moins deux locuteurs. En tant que tel, le sens est d'une part nécessairement commun et d'autre part pleinement linguistique, c'est-à-dire non-réductible à des phénomènes génétiques / biologiques ou téléologiques / fonctionnels. De cette manière de concevoir le sens il découle que la science du sens ne peut être fondée ni sur l'introspection (I'intuition) ni sur la perspective de I'observateur extérieur. Au contraire, la science du sens doit se faire à la fois interactionniste et empirique tout en étant basée sur la perspective de l'observateur participant » (1997 : 3, italiques de l'auteur). Nous adhérons intégralement à cette définition.

30. Pour une étude plus détaillée du système des articles, voir Douay \& Roulland (2014 : 162-168). On peut dire en anglais $\varnothing$ Water is cold! « cette eau / l'eau est froide ! » en sortant du bain pour désigner l'eau dont on sort aussi bien que $\varnothing$ Water is tasteless and colorless «L'eau n'a ni goût ni couleur » en général, ce dont le nom français est incapable. En français, le nom sans déterminant peut nommer mais ne peut pas désigner, sauf dans certains cas très contraints (certains cas de négation ou d'énumération, par exemple). Autre exemple de marqueur RID : l'auxiliaire DO en anglais (Douay \& Roulland 2016).

31. Voir notre analyse des prépositions SUR et SOUS en français dans Douay \& Roulland (2014 : 144-149).

32. Voir analyse d'autres marqueurs des différentes configurations dans Douay \& Roulland (2014: Ch. 6 à 9).

33. En anglais, l'usage est similaire. Voir l'exemple suivant avec a Mary who was hard as steel : Mary est présentée comme une personne double, avec une face connue (publique) et une autre face plus secrète et inquiétante : His wife... When he thought of his wife, he seemed to see two people. One the girl he had married, fair-haired, blue-eyed, gentle, reserved. That was the girl he had loved, the girl he teased whilst she stared at him with a puzzled frown. That was his Polly. But there was another Mary - a Mary who was hard as steel, who was passionate, but incapable of affection - a Mary to whom nobody mattered but herself. [Sa femme... Quand il pensait à sa femme, il lui semblait voir deux personnes. L'une était la jeune femme qu'il avait épousée, avec ses cheveux blonds et ses yeux bleus, gentille, réservée. C'était elle, la femme qu'il avait aimée, qu'il se plaisait à taquiner tandis qu'elle le fixait en fronçant les sourcils. C'était elle, sa Polly. Mais il y avait une autre Mary - une Mary aussi dure que le fer, pleine de passion mais incapable d'affection - une Mary qui ne se souciait que d'ellemême] (A. Christie, Ordeal by Innocence, Omniprose, 2003 : 216). 
34. Le terme de "disruption" signifie à la fois "rupture» et "renouvellement créatif ". Rappelant (pour ses propres préoccupations) la genèse du vocable, J.M. Dru (1996) insiste sur le fait que la disruption est " une méthodologie dynamique tournée vers la création ». Appliquée au domaine de l'économie, c'est "l'idée qui permet de remettre en question les "conventions" généralement pratiquées sur un marché pour accoucher d'une "vision", créatrice de produits et de services radicalement innovants». L'auteur souligne que "l'innovation disruptive est une innovation de rupture, par opposition à l'innovation incrémentale, qui se contente d'optimiser l'existant ». Il s'agit de « déclencher un (ou des) sursaut(s) créatif(s) [...] et de déboucher sur des innovations qui peuvent concerner aussi bien le produit lui-même, que le modèle d'affaires, le marketing ou la communication du groupe ".

35. Voir autres exemples, pour le français et l'anglais, dans Douay \& Roulland (2014).

36. Terme qui, dans notre théorie, est synonyme de « invariant » ou « valeur de base ». Le débat actuel sur l'invariance des formes tient à ce qu'on incorpore à la valeur systémique des données et paramètres contextuels au sens empirique ou situationnel. Autrement dit, il tient à l'absence de distinction claire entre système et usage et au refus ou à la méconnaissance, voire l'ignorance, de la notion de système.

37. Voir à ce sujet Douay \& Roulland (2014:20-24).

38. Il va de soi que, dans le cadre limité d'un article, il ne serait pas envisageable d'examiner un corpus plus ou moins exhaustif des phrasal verbs en collocation avec UP. Mais surtout une telle entreprise contredirait en grande partie une recherche de valeur systémique. Les études de corpus basées sur la fréquence concerne toujours pour nous des valeurs d'aval, contextuellement interprétées, et qui ont une pertinence strictement technique. L'objet de l'analyse linguistique systémique n'est pas de répertorier les usages de telle ou telle forme, mais d'expliquer le potentiel systémique dont cette forme est vectrice. L'invariant systémique permet ainsi d'expliquer non seulement les emplois attestés mais aussi ceux qui demeurent potentiellement attestables et que les relevés quantitatifs ne peuvent pas repérer.

39. On notera que, aussi bien pour slip up (interprété dans le contexte de cet exemple dans le sens «faire une rechute» alors qu'on était sur la voie de la guérison) que pour give up («baisser les bras, laisser tomber »), les traductions françaises pourraient davantage orienter vers DOWN que vers UP.

40. Dress up ici pourrait s'interpréter en rapport avec l'uniforme que porte la fille. Dans ce cas, la normalité ciblée n'est pas exactement la même : revêtir un uniforme est un cas de disruption. On change contextuellement d'angle de vue.

41. Voir infra pour d'autres interprétations de eat up.

42. La sous-spécification apparente de la particule est à apprécier précisément en fonction de son statut de relateur des partenaires communicationnels au contexte.

43. Stand up peut avoir d'autres interprétations. Voir stand up to someone "résister, tenir tête à quelqu'un ", It takes guts to stand up to her "Il faut du cran pour lui tenir tête ", stand up for oneself «avoir le courage de ses opinions, interposer son point de vue ».

44. Voir aussi upend au sens de "renversement d'un système ", upset «bouleversement ", upturn « retournement », uprise « révolte ", upheaval « insurrection ».

45. De manière amusante, mais symptomatique, le premier exemple donné par Linguee est give up , traduit par « baisser les bras ».

46. On dirait plutôt « aboutir à des conséquences opposées ».

47. On pourrait ajouter, pour rendre la nuance exprimée par UP, un adverbe comme «malencontreusement».

48. Selon la formule courante utilisée par les psychologues à propos des crises que peut traverser un couple : « a break is not a break up ». À propos d' « état » et de rupture, on pourra se reporter à la très fine analyse de l'imparfait français par Desclès (2000). Dans notre modèle, l'imparfait est précisément une forme $\mathrm{C} 1$ disruptive. 
49. On peut penser à sign up qui n'est pas seulement " apposer sa signature », mais " prendre fait et cause » pour quelqu'un ou quelque chose, ou pour un mouvement collectif.

50. On notera que UP dans cette expression est le plus souvent placé après le complément (one's socks), ce qui permet une insistance sur le sens de «mobilisation » dont UP est vecteur. Ce qui montre bien qu'il faut dissocier le sens de UP du sens du verbe lexical qu'il ne «complète » pas simplement ni par rapport auquel il serait redondant.

51. Le sens latin premier du préfixe RE- est « en arrière » et non pas, comme le définirait une sémantique de l'évidence, « à nouveau ». « Remonter les bretelles à quelqu'un », c'est le remettre à sa place, celle qu'on lui reproche d'avoir quittée ; dans « revenons à nos moutons ", il y a une incitation à revenir en arrière, au point qu'on avait abandonné provisoirement lors d'une digression, et non pas une simple reprise. Voir à ce sujet P. Jalenques (2002), I. Weill (2009), et les nombreux exemples analysés par F. Nemo (2014) comme «se replier", ou «repousser", « rechigner». Dans ces exemples, ce qui est signifié, c'est une différenciation (opposition) par rapport à un cours des choses (F. Nemo, dans un cadre théorique très différent, parle pour sa part de mouvements P1 et P2 anti-orientés).

52. La traduction Robert Laffont 2019 (de Cécile Arnaud, Jean-Daniel Brèque, Odile Demange, Nathalie Gouyé-Guilbert et Dominique Haas) est la suivante : "Le père et le fils étaient de petite stature, l'un comme l'autre, ce qu'ils cherchaient à compenser par un naturel jovial et blagueur » (c'est nous qui soulignons).

53. Les recherches vont fréquemment dans le sens de cette problématique. Par exemple, le concept psychologique d'affordance (Gibson) vise à automatiser dans l'organisme certains éléments de perception, remettant en selle une forte contribution de l'environnement à la structuration. Dans notre analyse, l'environnement est a priori complexe et ne se laisse approcher (afford) qu'en fonction des latitudes que le système déjà constitué offre à l'organisme.

\section{RÉSUMÉS}

Cet article souhaite montrer que les modèles spatiaux, physiques ou expérientiels, proposés généralement pour rendre compte du fonctionnement des particules verbales en anglais, posent de nombreux problèmes. En particulier, il s'avère impossible de réduire le signifié de UP (vs DOWN) à l'expression d'un «mouvement vertical vers le haut » et les valeurs non spatiales sont très nombreuses. L'alternative décrite ici, dans le cadre général de la Théorie de la Relation Interlocutive (Douay \& Roulland 2014), consiste à rechercher dans le fonctionnement même du système linguistique les causes et les motifs à la fois de la structuration spatiale et non spatiale. Cette recherche s'appuie sur une définition systémique du langage fondée sur l'auto-référence et l'auto-organisation, plutôt que sur des stimuli environnementaux qui seraient plus ou moins « évidents ».

Our aim in this article is to point out that mainstream spatial models (either world-based or experience-based) prove largely inadequate for the analysis of verbal particles in English. In particular the particle UP (vs DOWN) definitely cannot be confined to simply expressing "upward movement" and non-spatial uses are very frequent. We propose here an alternative model for UP in the general framework of the Theory of the Interlocutive Relation (Douay \& Roulland 2014), based on systemic operations of language which can account for spatial and non-spatial structural patterns as well. The key-concept in this research is a systemic definition of language based on 
self-reference and self-organization rather than more or less "obvious" environment-induced stimuli.

\section{INDEX}

Keywords : UP, DOWN, verbal particle, spatial model, system, systemic, self-reference, theory of the interlocutive relation

Mots-clés : UP, DOWN, particule verbale, modèle spatial, système, systémique, auto-référence, théorie de la relation interlocutive 